\title{
A COMPUTER-AIDED TELESCOPE POINTING SYSTEM UTILIZING A VIDEO STAR TRACKER
}

James P. Murphy, Kenneth R. Lorell, and Charles D. Swift

Ames Research Center

Moffett Field, Calif. 94035

(NASA-TM-X-73079) A COMPUTER-AIDED

TEIESCOPE POINTING SYSTEM UTILIZING A VIDEO

STAR TRACKFR (NASA) $55 \mathrm{p}^{-}$CSCL 0.3A

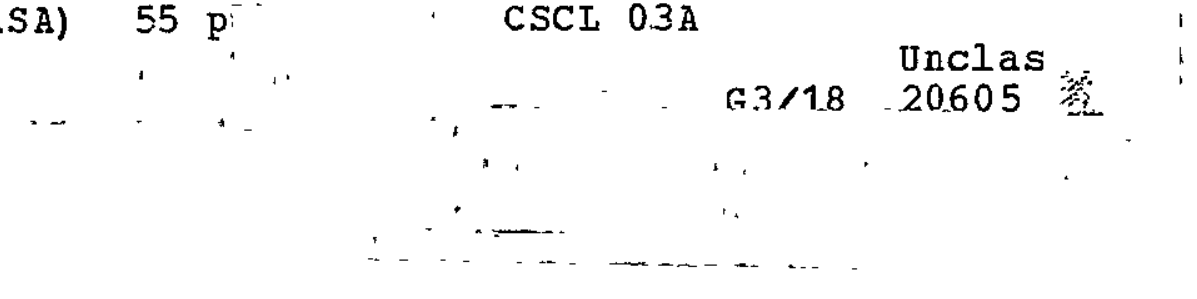

December 1975 


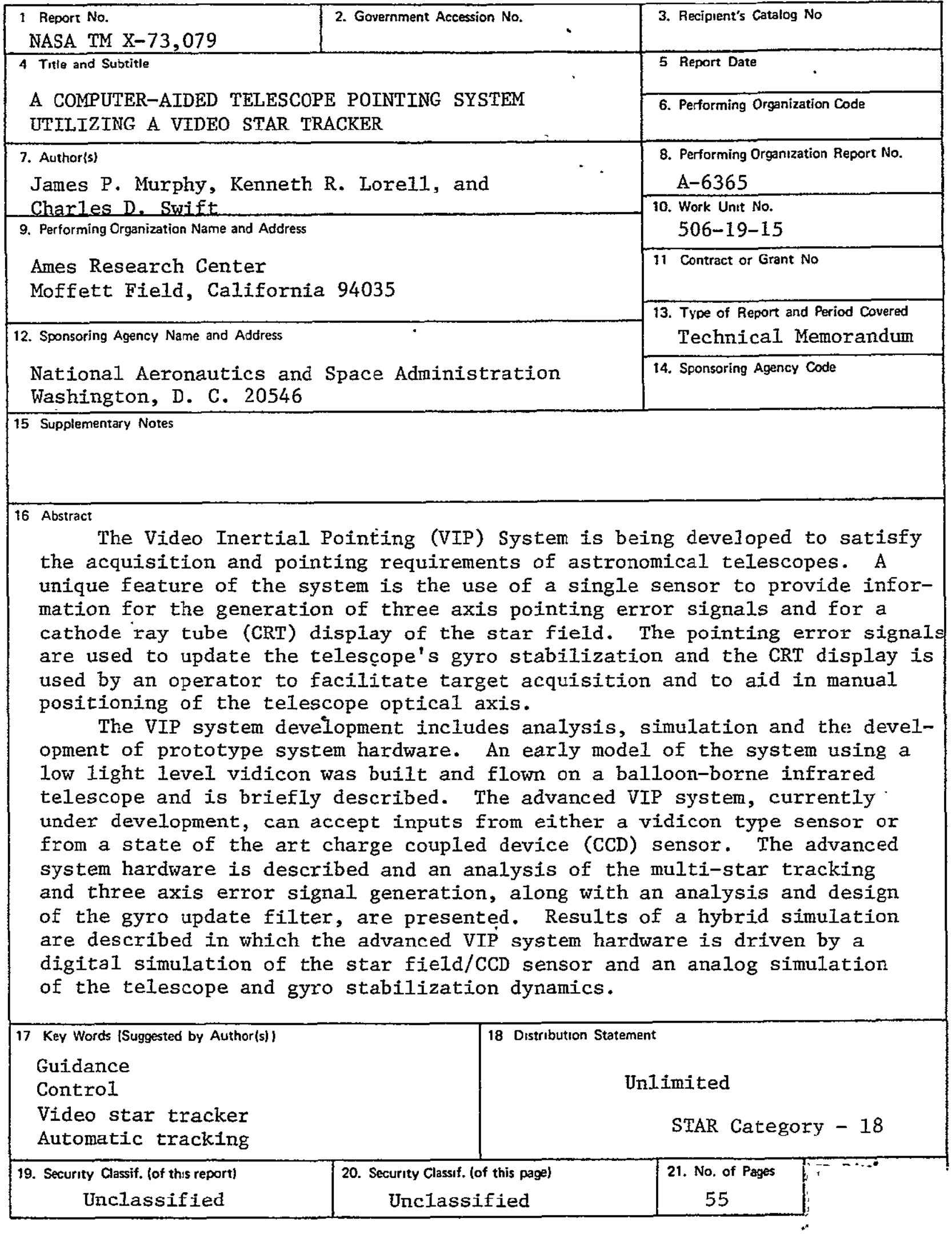

-For sale by the National Technical Information Service, Springfield, Vırgınia 22161 


\section{SYMBOLS}

a

C

$\mathrm{C}_{i j}$

D

$d_{G}$

F

G

H

J

K

$\mathrm{K}_{\mathrm{P}}, \mathrm{K}_{\mathrm{L}}$.

$\mathrm{K}_{x x}, \mathrm{~K}_{x y}, \mathrm{~K}_{y x}, \mathrm{~K}_{y y}$

$\mathrm{K}_{1}, \mathrm{~K}_{2}$

L

$\mathrm{M}_{\mathrm{v}}, \mathrm{M}_{\mathrm{C}}$

$\cdot n$

${ }^{n} \mathrm{G}$

$Q_{i}$

$\mathrm{S}$

$\mathrm{T}_{1,2}$

I

T

${ }^{W_{P}, \mathrm{Y}, \mathrm{Rij}}$

x, $x_{j}$

$\underline{x}_{\mathrm{n}}$

$x_{i}, y_{i}$

$\mathrm{x}_{S_{i}}, \mathrm{y}_{S_{i}},{ }^{z_{S}}$

$\mathrm{x}_{\mathrm{B}_{i}}, \mathrm{y}_{\mathrm{B}_{i}}, \mathrm{z}_{\mathrm{B}_{\mathrm{i}}}$
CCD well size

transfer fon of telescope controller: direction cosine matrix

elements of direction cosine matrix

demodulator transfer function.

gyro drift

plant dynamics matrix

gyro transfer function: control distribution matrix

gimbal torque motor transfer function

telescope/gimbal inertia along control axis

scale factor system gain constant

discrete filter gains

augmented control gains

continuous filter gains

update filter transfer function

visual magnitude

step number in discrete system: number of tracked stars

gyro noise

charge in CCD well

La Place variable

delay times in star tracker and computer

integration time of star tracker.

sample period

weighting function

state vector, system state variable

discrete system state vector

CCD location of star i

inertial coordinates of star i

body coordinates of star i 
$\Delta \mathrm{x}_{i}, \Delta \mathrm{y}_{i}$

$\mathrm{x}_{\mathrm{F}}, \mathrm{y}_{\mathrm{F}}$

$\mathrm{y}_{\mathrm{n}}$

Z

$\Gamma, \Gamma_{y}$

$\phi, \theta$

$\Phi, \Phi_{\mathrm{y}}$

$\theta_{\mathrm{S}}$

$\theta_{\mathrm{Sn}}$

${ }^{\theta} \mathrm{P}, \mathrm{Y}, \mathrm{R}$

$\theta_{G}, \theta_{G_{0}}$

$\sigma$

$E\{\}$

^

$-$

(S)

(Z)
CCD position errors for star $i$

star tracker field of view coordinates

discrete filter output

Z-transform variable

control distribution matrices

right ascension and declination of a star

state transition matrices .

star tracker attitude error

discrete star tracker attitude error

three axis attitude error's

gyro output, initial angle

standard deviation

expected value

estimated value

average value

La Place Transform

Z-transform

differential with respect to time 


\section{A COMPUTER-AIDED TELESCOPE POINTING SYSTEM UTILIZING A VIDEO STAR TRACKER}

James P. Murphy, Kenneth R. Lorell, and Charles D. Swift

Ames Research Center'

INTRODUCTION

Development of the Video Inertial Pointing (VIP) System was initiated at the Ames Research Center in 1973. The system concept and development has its origins in infrared (IR) astronomy investigations currently underway and the concept includes features which anticipate the acquisition and pointing requirements for astronomical telescopes of the Space Shuttle era.

The initial version of the VIP system was developed to satisfy the acquisition and pointing requirements of a balloon-borne IR telescope. This system used a conventional SIT vidicon boresighted to the telescope to provide information for a cathode ray tube (CRT) display at the ground station operators console and information to update the telescope's gyro stabilization. An operator at the control console completed acquisition manually using a joystick and the CRT display. He then was able to select a single guide star for use in automatic on or off axis tracking. For this paper, on axis tracking refers to tracking a visible target on the common optical axis of the video sensor and telescope. Off axis tracking refers to tracking a visible target in the field of view (FOV) of the video sensor but not on the common optical axis. This system was used for several balloon flights and proved to be very useful.

Because of the value of the early system, it became apparent that the concept had application to the more advanced airborne and Space shuttle telescopes, such as the one meter class cryogenically cooled IR telescope being considered as a shuttle payload (ref. 1)\% Consequently, a program was initiated to develop the VIP system to satisfy the requirements of these more advanced applications. Requirements of these telescopes include precise three axis pointing, manual and automatic modes of operation, and an interface with more' complex and precise gyro stabilization systems.

Key aspects of the advanced VIP system include use of a state-of-the-art charge coupled device (CCD) video sensor which provides the capability for precision pointing; multi-star processing algorithms to generate the three axis pointing error signals and a.gyro update filter which combines the video and gyro sensor data. The analysis and development of the multi-star processing and gyro update filter are presented along with some system tradeoffs that must be considered for design of an operational system. A prototype of the advanced system has been constructed and tests of this hardware with a hybrid simulation of the CCD sensor and telescope dynamics verify the system design and performance. Laboratory tests with a prototype CCD sensor have been conducted and the results are used in the analysis and hybrid simulation (ref. 2). Although not reported in this paper, the prototype CCD sensor will 
be used with the VIP system hardware on a ground based gimbal and gyro stabil- . ization system for complete system hardware tests.

It should be noted that the VIP system does not include design or deve1opment of the gimbal and gyro stabilization. VIP is intended to interface with this hardware and consequently, the design aspects of the gimbal and gyro stabilization are not discussed in this report. Similarly, for telescopes that require ultra precise pointing accuracy (below 1 arc sec) or that have significant movement of the optics with respect to the outer telescope structure, an internal fine guidance sensor is required. Although this report only treats a system using an external sensor boresighted to the telescope, the techniques can be applied to a system using a video sensor for fine guidance.

The authors gratefully acknowledge the assistance of Messrs. W. F. Barrows of NASA/ARC, J. K. Lee of the Informatics Co., and A. J. Throckmorton of Stanford University in developing the VIP hybrid simulation and the many hours spent making it operational.

CONCEPT OF VIDEO INERTIAL POINTING

Various approaches have been used in the past to provide the target acquisition and stabilized pointing for astronomical telescopes (ref. 3 ). The rotation of the earth and its stability are used by ground-based telescopes with a knowledge of the target location in the celestial sphere. A "finder" scope allows the astronomer to observe the star scene and to make final adjustments to the telescope angles and drive rates. For nongroundbased telescopes where operation is remote, gyroscopes are used as the primary inertial reference or stabilization and image disector type star trackers are employed to correct for inherent gyro drift. Typically, several of these star trackers are used (at least two to provide three axis pointing information) but they cannot provide simultaneous pointing error information and information for a star field display. Consequently, conventional systems for balloon, air- and space-borne telescopes do not provide the same interaction and control available to the ground-based astronomer.

The Video Inertial Pointing System takes advantage of recent improvements in video sensor and electronics technology to provide a telescope pointing system with fewer sensors than the conventional system and one that allows a more direct interaction with the control through a star field display at a control console. The system uses both gyro-stabilization and a video type star tracker. Primary stabilization is provided by conventional inertial

techniques. The telescope remains stabilized, within performance tolerances of the gyros and servo electronics, in the inertial coordinate system. Fine pointing and control are made possible using star field information from the 
video sensor attached (usually boresighted) to the main telescope. The guide star locations from the video sensor are compared with the previously-stored locations of these same stars. Differences between the current and desired Iocations of these stars causes the system to generate error signals that are used to update the gyro outputs. The telescope (and video sensor) thus move to a vector so that the desired and current locations of the guide stars coincide. Since the system can be made to come to a stabilized null with the guide stars at any desired location in the field of view, it can be used to track any point in the field of view even if there is no visible star at that point (i.e., off-axis pointing). In addition, the system has information from more than one star so that it can provide three axis pointing control using only a single video sensor.

Another feature of the VIP system is the star field presentation avai1able to the observer/operator. Since the star sensor is of the video type, it is only necessary to present the output on a CRT display. In contrast, the conventional star tracker must resort to electronic or mechanical scanning in order to build up comparable star field information. It is not possible to provide simultaneous pointing error signals and star field display information. In the systems described in this report, direct high bandwidth video is not used to drive the CRT display. Rather the video sensor data is processed to allow more elaborate display options (in the case of the advanced system) and to reduce the bandwidth for acceptable transmission (in the case of the first generation system discussed in the next section).

\section{FIRST GENERATION SYSTEM}

A first generation model of the Video Inertial Pointing System has been completed and integrated into a balloon-borne telescope system for testing and evaluation. Figure $I$ is a block diagram showing the key elements of the system.

The telescope is gyrostabilized in two control axes perpendicular to the telescope line of sight, elevation and cross elevation. Mounted on the telescope and boresighted to it is a conventionally scanned silicon intensified target (SIT) vidicon camera. Its $75 \mathrm{~mm} \mathrm{f} / 1.4$ lens results in a field of view of approximately $7^{\circ} \times 10^{\circ}$ and sufficient sensitivity to track +6 th magnitude stars. Star field video and synchronization pulses from this camera are fed to on-board processing electronics consisting of a video conditioner, gate generator and coincidence detector. The video conditioner circuit generates a timing pulse for each star signal above a commandable threshold, and in addition, generates $\mathrm{x}, \mathrm{y}$ coordinate signal's and intensity signals for up to ten stars. These values are telemetered to the ground station CRT display. The gate generator uses the TV synch pulse to generate four timing gates which can be moved (by telemetered command) relative to the synch pulse so that they can be positioned to surround any location in the video format. The duration of the gates can be varied to include as few as 5 or up to 64 video scan lines. The square dead area surrounded can be varied from 2 to 20 video scan lines. Star timing pulses and gate signals are fed to the coincidence circuit. If a 
timing pulse is coincident with one of the gate signals then an appropriate output signal is coupled to the gyro servo system which moves the telescope in the correct direction to move the star's timing pulse into the dead area in the center of the timing gate pattern.

Control of the tracking system is by commands telemetered from the ground station. The operator or observer views the star field on the CRT display. Intensified cross hairs are visible on the display as well. The location and size of these cross hairs indicates the location and size of the timing gates in the video format. By manipulating a joystick, the cross hairs can be moved to cause a coincidence with the desired star. The on-board system will then move the telescope to put the star in the center of the pattern. Further movement of the cross hairs is then possible to put the tracked star at whatever location is required in the field of view of the TV camera.

Since this first system has only two axes of stabilization, periodic adjustments to the tracking are required to correct for diurnal motion. These adjustments are only necessary when the tracked star is not at the center of the field of view (off axis tracking). During ground-based tests and two flight tests this system performed very well. Although the tracking accuracy depends on whether the guide star is on axis or off axis, the object of interest was within one arc minute of the optical axiș of the telescope for extended periods of time during typical operation.

\section{ADVANCED SYSTEM DESC̈RIPTION}

The advanced VIP system consists of three primary subsystems; a video sensor whose optical axis is coaligned with the telescope optical axis, an electronics package which includes a microprocessor and interface electronics, and a control console with CRT display from which the system is operated. Figure 2 depicts, the inter-relationship between the telescope and VIP systems. In this section a description of the VIP components will be given, followed by a discussion of system operation.

The video star tracker utilized by VIP is a key element in the system. Two important features of VIP are dependent on the video sensor; the ability to provide three-axis control with only a single star tracker, and a CRT display of the telescope FOV for the instrument operator. The advanced VIP system is designed to accept inputs from two types of sensors, either a conventional low-light level camera utilizing an Intensified SIT (ISIT) vidicon tube, or a state-of-the-art CCD camera which is all solid state and incorporates its own microprocessor. While the ISIT sensor is a proven device and embodies relatively little technological risk, it does require an additional interface to the VIP computer in order to provide the required digital input format for multiple star tracking. The CCD tracker, in addition to the benefits accruing to an all solid-state device, has remarkable flexibility and is ideally suited to the VIP system. Both types of sensors employ interpolation techniques in order to obtain improved resolution. The $\mu$-processor in the CCD sensor electronics contains an algorithm to do digital interpolation. 
The ISIT camera, interface electronics also contains a $\mu$-processor in order to perform the interpolation and data formatting functions.' A more detailed. description of the CCD tracker and its operation is presented in a later section.

The VIP electronics package has two main sections, a National Semiconductor IMP-16 $\mu$-processor and a group of circuits which interface the $\mu$-processor to the star tracker, telescope control system, and VIP control console. Figure 3 is a block diagram of the VIP electronics.

The $\mu$-processor plays a central role in the VIP system. In addition to converting star tracker data into three-axis error signals via the multistar processing algorithm, it performs all of the logic sequences necessary to initialize and track, it drives the CRT display, and interrogates VIP control console switches to perform operator-controlled functions. The VIP $\mu$-processor also communicates directly with the star-tracker $\mu$-processor in order to effect data transfer as well as control the integration time and threshold level of the tracker. Figure 4 is a flow chart of the program used by the $\mu$-processor.

In general, the entire electronics subsystem is designed to minimize hardware and place as many logic, data manipulation, and sequencing functions in software as possible. Thus, the primary function of the circuitry surrounding the IMP-16 in figure 3 is to control input/output. Inputs from the control panel are detected by two jump-condition lines fed by a multiplexer which decodes the device address. Star-tracker data is clocked directly into the 16-bit data bus. Outputs are handled by 8-, 12-, and 16-bit latches which store the data when their appropriate addresses are decoded. CRT display requirements dictated that a special buffer be used to store video-output data. One 12-bit D/A is used to drive analog circuitry by storing its output in sample and hold circuits selected in the correct sequence by the address decoder. Additional circuits are used for switching, impedance matching, and filtering.

The VIP control panel and CRT display are shown in figure 5. The operator controls the position of the telescope LOS with the joystick and may observe a variety of star-field presentations on the CRT display. Switches controlling VIP operation, auto/manual select, fast/slow slew rate, and star field display options, surround the joystick controller. Threshold level and integration period for the star tracker are selected by means of two thumbwheel switches on the operator's left.

Other functions, such as automatic scan, are controlled from switches on the operator's right. Although the prototype equipment has an IMP-16 which is programmable with paper tape, an operational version would use a $\mu$-processor with PROM program storage and would be co-located with the rest of the electronics package.

Operation of a VIP-controlled telescope begins with coarse acquisition of the target star field. This is accomplished by slewing the telescope to a precomputed orientation and making an initial alignment with the joystick 
controller in the manual mode. The star field, as viewed by the video sensor, is displayed on the CRT and the operator orients the telescope by comparing the CRT picture with a star chart. Alternatively, a computer-stored star map of the desired viewing area may be presented. When the target has been identified, the automatic mode is selected by pushing the "initialize" button located on the end of the joystick. This commands the VIP . store the $x-y$ locations of all of the stars being tracked by the video

- sensor. Each time the current star locations are transmitted to VIP by the star tracker, the pointing error in all three axes is calculated by comparing these current star positions with those stored in memory upon the "initialize" command. The calculated attitude error and the gyroscopically measured attitude error are then combined in the $\mu$-processor gyro update filter to provide the torque commands fed back to each of the three gyros..

The operator may continue to reposition the telescope optical axis during automatic operation. A display option may be selected in which only the star field in its desired location is presented. Joystick activation moves this pattern on the CRT screen to whatever new position is desired. The dynamics of telescope motion are not seen (since only commanded star field position is displayed) thereby enabling very precise positioning. Simultaneous selection of current telescope position for the display will indicate to the operator how close the telescope is pointing to the desired location. The CRT will show two star fields in this mode, a bright one for actual position and a dimmer field for the desired position. When the two fields are coincident, the telescope is on target.

A digital indicator on the control console informs the operator of the number of stars currently being used for tracking. Another display option permits viewing only these stars. As new stars enter or depart the field of view, VIP has the capability to display them without disturbing the tracking function, as well as continually keeping the operator informed of the number and location of tracked stars. If, because of slewing or tracker parameter changes, the number and/or position of tracked stars is unsatisfactory, the operator may reinitialize the system by pressing the "initialize" button again.

VIP is designed to permit maximum operator interfacing with the automatic control system and provides him with several display options, indicators, and controls so that precise orientation of the telescope becomes a simple task.

\section{CCD SENSOR DESCRIPTION}

The advanced VIP system is designed to use either an ISIT vidicon camera or a sensor employing a charge coupled device (CCD) detector. Although conventional sensors do provide adequate performance, as demonstrated in the first generation system, there are a number of advantages, particularly for a digital system, that accrue from the use of a CCD sensor. Some of these will. be discussed below, followed by a description of CCD sensor operation. 
The primary advantage of a CCD sensor, compared with trackers utilizing vidicon or image dissector tubes, is the elimination of the scanning electron beam and the accuracy with which digital position information for multiple stars is generated. The absence of the drive circuitry, electron gun, control yoke, and other components associated with a scanning beam device is a considerable simplification in star tracker technology. Since the sequencing, control functions, and computation in VIP all rely on a digital computer, the inherent digital nature of a CCD sensor means the elimination of an analogto-digital interface giving a considerable savings in system complexity. In addition, since all components are solid state, there are no high voltages, the operational life and reliability are enhanced, and the weight, size, and power all reduced.

The face of the CCD detector is divided into a rectilinear pattern of discrete photosensitive elements, each connected to readout registers also located on the front surface. As photons strike the detector, the charge built up in each element is transferred to the readout registers after a selectable period of integration, the charge being directly proportional to the number of photons striking an element during the integration period. Since the location of each element is uniquely specified, it is possible to obtain'the precise coordinates, within the resolution of the photosensitiveelement matrix, of any stars being imaged on the surface of the CCD.

The current state of the art for CCD's is a matrix of about 400 elements square. Commercially available CCD's are about 200 elements on a side. Thus, even the most advanced units cannot obtain resolution better than 0.5 arc min for $3^{\circ}$ FOV optics. However, it is possible through a process of interpolation to improve the resolution by an order of magnitude (ref. 2).

The interpolation scheme requires that the output of a four by four matrix of elements be sampled and averaged each time the CCD is scanned. This array is centered on the element which first exceeds a pre-selected threshold during the initial scan of the device. Thus, for each star image, there is a corresponding four by four matrix for which the charge "center of mass" is computed. It is possible, therefore, to obtain computed star locations which are not centered on an element. The effect of this procedure is to increase the resolution by artificially increasing the element density.

The interpolation algorithm and the data formatting for use by the control system are of a complex nature and require extensive data handling for their implementation. This is accomplished through the use of a microprocessor built into the electronics of the star tracker. Raw data from the CCD is read at the end of each integration period by the microprocessor which then computes the locations of the ten brightest stars in the field of view. 'Each star is assigned an identification number and the four pieces of data for each star ( $x, y$, brightness, i.d.) are transferred to the VIP computer when the star tracker microprocessor completes the data for all of the stars in a frame. Logic within the microprocessor automatically takes care of stars entering or leaving the field of view so that while it is always possible to display all 
stars in the field of view, the VIP controller only accepts data for valid stars. ${ }^{1}$ Initializations of the star tracker may be accomplished at any time at the option of the operator via a pushbutton located on the VIP control stick.

The integration period and threshold are also controlled by the star tracker microprocessor. Seven levels of both functions are selectable from thumbwheel switches on the VIP control panel. These levels are arranged so that each increment of integration time changes the sensitivity by $0.25 \mathrm{M}_{\mathrm{v}}$ and each threshold increment changes the sensitivity by $1.0 \mathrm{M}_{\mathrm{v}}$. Thus, if

$$
Q_{i}=\mathrm{KT}_{j}\left(2.5118^{-\mathrm{M}_{\mathrm{v}}}\right)
$$

describes the charge built up in a given element during an integration period $j$, and we desire to have a $+0.25 \mathrm{M}_{v}$ change for each increase $\mathrm{T}_{j}$, then

$$
\begin{aligned}
Q\left(T_{j}, M_{v}\right) & =Q\left(T_{j+1}, M_{v}+0.25\right) \\
T_{j+1}\left(2.5118^{-\left(M_{v}+0.25\right)}\right) & =T_{j}\left(2.5118^{-M_{v}}\right)
\end{aligned}
$$

or

$$
\mathrm{T}_{j+1}=1.2588 \mathrm{~T}_{j}
$$

For a given $T_{j}, Q_{i}\left(T_{j}\right)$, the threshold below which a star will be rejected a's being too dim, may be expressed as

$$
Q_{i+1}\left(T_{j}\right)=(2.5118)^{-1} Q_{i}\left(T_{j}\right)
$$

From equations (2) and (3) we have a method of computing the appropriate integration times and threshold levels given the minimum exposure time, system gain constant $\mathrm{K}$, and the maximum desired $\mathrm{M}_{\mathrm{v}}$. For practical considerations, $\mathrm{T}_{\mathrm{j}_{\min }}=0.250 \mathrm{sec}$ and $\mathrm{M}_{\mathrm{v}_{\max }}=8$. It is important to note that the relationship between the actual integration times or threshold levels and the positions of the thumbwheel switches on the control panel are arbitrary, the star tracker microprocessor need only use the switch position code to locate the desired value of integration time or threshold in a look-up table stored in memory.

Table 1 lists the values of $Q_{i}\left(T_{j}\right)$ and the range of $M_{v}$ (brightest and dimmest stars) for any combination of threshold and integration time. $K$ has been arbitrarily set to $10^{3}$.

${ }^{1} \mathrm{~A}$ valid star is one that has met a number of tracking criteria, both in the star tracker and within the VIP software. Attitude information is generated using only valid stars. 
Figure 6 is a view of the star tracker cut-away to show the major components. A modular approach has been taken to the optical and mechanical design so that the tracker may be easily modified to fit a variety of mission requirements. A Peltier-effect cooler is included for CCD operation on the ground; operation of the cooler during high altitude or orbital flight is not required.

VIP GYRO FILTER ANALYSIS

A number of investigators have approached the problem of combining star tracker information and gyro output to improve inertial orientation. Farrenkopf (ref. 4) uses a precise gyro error model with pre- and post-update filter error analysis to construct a family of curves which predict the performance of systems of this type. Rauch (ref. 5) has analyzed the errors in a singleaxis star-tracker/gyro system. He obtains the rms steady state pointing error as a function of the number and accuracy of star measurements and the accuracy of the gyro. Potter and Vander Velde (ref, 6) derive an analytical solution to the problem of combining gyro and star tracker data in an optimal fashion.

In all of the work described above, the authors have investigated the interrelationship between high bandwidth, high (relatively) noise gyros and low bandwidth, low noise star trackers when their outputs are combined for inertial stabilization. However, the problem of design, and in particular the interfacing of digital and continuous systems, has not been addressed. In this section the design of a simple filter for the VIP system is discussed. and the results of an analytical technique which utilizes computer-generated 2 transforms is presented.

Figure 7 is a block diagram illustrating the basic elements of a gyrostabilized telescope controller to which a VIP system has been added. The telescope control loop is closed by coupling the output of a single axis rate integrating gyro to a torque motor through a conventional compensation network. The system has excellent transient response and, with integral control, has good performance even when a body-fixed disturbance torque is applied. The gyro output is updated by reference to the attitude error measured by a star tracker.

The Separation Theorem implies that the operation (i.e., pole placement) of the update filter $L(S)$ will not affect the plant controller as long as the filter model reflects the plant dynamics. Thus, if a continuous measurement of attitude error were provided, the design of the gyro update filter could proceed along the lines of classical control theory. For example, since the goal of the controller is to use $\theta_{G}$ to force $\theta=0$ and that of the filter is to use $y$ to force $\theta_{G}=0$, then

$$
\theta(S)=-\frac{H C\left[d_{G}(S) G D+\theta_{G O}\right]}{J S^{2}(1+L G D)+\operatorname{HCGD}(S+L)}
$$




$$
\theta_{G}(s)=\frac{\left[d_{G}(S) G D+\theta_{G o}\right] J S^{2}}{J S 2(1+L G D)+H C G D(S+L)}
$$

for a simple gyro approximation, $G D=\frac{1}{S}, d_{G}(S)=\frac{d_{G}}{S}$ so.

$$
\begin{aligned}
& \theta(\mathrm{s})=-\frac{\mathrm{HC}\left(\frac{\mathrm{d}_{\mathrm{G}}}{\mathrm{S}}+\theta_{\mathrm{Go}} \mathrm{s}\right)}{\left(\mathrm{JS^{2 }}+\mathrm{HC}\right)(\mathrm{s}+\mathrm{L})} \\
& \dot{\theta}_{G}(s)=\frac{J\left(d_{G} s+{ }_{G o} s^{2}\right)}{\left(J S^{2}+H C\right)(S+L)}
\end{aligned}
$$

thus equations (6) and (7) imply a simple filter of the type $L(S)=K_{1}+\frac{K_{2}}{S}$ will eliminate $d_{G}$ and $\theta_{G_{0}}$. An alternate method of arriving at the same form is to mechanize an observer for $\theta_{G}$ using $\theta_{S}$ as a measurement. Then the system dynamics are

$$
\begin{aligned}
& \dot{\theta}_{G}=\mathrm{d}_{G}+\dot{\theta} \\
& \dot{\mathrm{d}}_{G}=0
\end{aligned}
$$

and for an observer

$$
\begin{aligned}
& \dot{\hat{\theta}}_{G}=\dot{\hat{d}}_{G}+K_{1}\left(\theta_{S}-\hat{\theta}_{G}\right)+\dot{\theta} \\
& \dot{\hat{d}}_{G}=K_{2}\left(\theta_{S}-\hat{\theta}_{G}\right)
\end{aligned}
$$

Equations (9) lead to the same form of filter as before. Although typical systems of the type shown in figure 7 have 10 or more states, the procedure of selecting $\mathrm{K}_{1}$ and $\mathrm{K}_{2}$ is a straightforward task for a linear system.

The use of a digital star tracker and digital computation to determine the attitude error introduces a number of elements into the system which have a direct, and adverse, effect on the performance. These include sampling, time delays in both the star tracker and computer, mixing of delayed and nondelayed data, and quantization. Thus, a major difficulty is introduced into the analysis and design of a system with these characteristics. The update filter couples into the stabilization loop dynamics and unstable operation of the two loops will result if the gains $K_{1}$ and $K_{2}$ are selected without properly taking into account all of the dynamical effects present.

Classical S-plane analysis is still possible for a system with sampling and time delays if some form of polynomial representation of $e^{-\mathrm{ST}}$, such as the Padé approximant ( $r e f .7$ ), is used. This technique is mathematically cumbersome, however, and provides relatively imprecise knowledge of the gainresponse interaction. An alternative is the Z-transform which is ideally suited for the analysis of problems of this type, but has the disadvantage of 
being difficult to calculate, especially for systems with a large number of states. This problem has been circumvented through the use of computer programs (ref. 8) which calculate the Z-transform, and find the roots of the resulting characteristic equation.

A two-step process is used to obtain the $\Phi$ and $\Gamma$ matrices of the complete system. First the continuous portion of the system, without tine delays or digital filter, is transformed. Augmented state transition and control distribution matrices may now be formed by adding the appropriate Z-transforms for the time delays and digital portion of the control (filter). Thus, for step one:

$$
\underline{\dot{x}}=\mathrm{F} \underline{\mathrm{x}}+\underline{\mathrm{Gu}} \rightarrow \underline{\mathrm{x}}_{\mathrm{n}+1}=\Phi \underline{\mathrm{x}}_{\mathrm{n}}+\underline{\Gamma}_{\mathrm{u}}
$$

and for step two:

$$
\left[\begin{array}{c}
\mathrm{x}_{\mathrm{n}+1} \\
\mathrm{y}_{\mathrm{n}+1}
\end{array}\right]=\left[\begin{array}{ll}
\Phi & 0 \\
0 & \Phi_{\mathrm{y}}
\end{array}\right]\left[\begin{array}{l}
\mathrm{x}_{\mathrm{u}} \\
\mathrm{y}_{\mathrm{n}}
\end{array}\right]+\left[\begin{array}{cc}
\Gamma & 0 \\
0 & \Gamma_{\mathrm{y}}
\end{array}\right]\left[\begin{array}{cc}
\mathrm{K}_{\mathrm{xx}} & \mathrm{k}_{\mathrm{xy}} \\
\mathrm{k}_{\mathrm{yx}} & \mathrm{K}_{\mathrm{yy}}
\end{array}\right]\left[\begin{array}{c}
\mathrm{x}_{\mathrm{n}} \\
\mathrm{y}_{\mathrm{n}}
\end{array}\right]
$$

Z-plane plots of the eigenvalues of equations (10), analogous to closed loop root loci in the S-plane for a continuous system, permit the designer to select filter gains to optimize whatever criteria he desires. In addition, the changes in performance as a function of the time delays may be observed and corrected for. In particular, $T_{1}$, the CCD integration time, has a wide dynamic range.

Figure 8 is a state variable representation of the system shown in figure 7 with numerical values for a gyrostabilized telescope used at the Ames Research Center. This system, with appropriate values for the time delays and sampling intervals, has been analyzed. A filter similar in form to the one discussed for the case of a linear system was implemented in the form of the difference equation

$$
\mathrm{y}_{\mathrm{Gn}}=\mathrm{y}_{\mathrm{G}(\mathrm{n}-1)}+\left(\mathrm{K}_{1}+\mathrm{K}_{2}\right)\left[\theta_{S(n-1)}-\theta_{G(n-1)}\right]-\mathrm{K}_{1}\left[\theta_{S(n-1)}-\theta_{G(n-1)}\right]
$$

$L(Z)$, the Z-transform of the filter is

$$
L(Z)=K_{L} \frac{\left(z-K_{P}\right)}{(z-1)}
$$

where

$$
\mathrm{K}_{\mathrm{L}}=\left(\mathrm{K}_{1}+\mathrm{K}_{2}\right), \quad \mathrm{K}_{\mathrm{P}}=\frac{\mathrm{K}_{1}}{\left(\mathrm{~K}_{1}+\mathrm{K}_{2}\right)}
$$

Figures 9 and 10 represent a series of typical Z-plane loci calculated for this system. The effects of gain changes and variations in the delay times are quite evident. The general effects of the time delays is to strongly increase the sensitivity to changes in $\mathrm{K}_{\mathrm{I}}$ and require much smaller 
values of $K_{2}$. A natural result is that speed of response of the entire system is considerably lessened and transient behavior is degraded.

A conclusion to be drawn from this analysis is that a more sophisticated filter mechanization which models the time delays is required in order to improve performance. While our prototype system utilizes the simple filter described above, investigations are currently under way into the analysis, design, and implementation of filters which incorporate a more precise plant mode1.

\section{VIP MULTI-STAR PROCESSING}

To determine the attitude error signals needed to point a telescope, at least two guide stars must be used. If a single guide star were used pointing information for two axes perpendicular to the telescope's line of sight could be generated. Gyro information, subject to drift, would then be required to orient the telescope about its optical axis. Since the video sensor used with the VIP system provides position information for up to 10 guide stars, the complete three axis pointing errors needed to point the telescope, as well as error signal averaging used to eliminate the effects of video sensor noise and inaccuracies, can be generated.

Appendix A contains a derivation of the basic equations relating the desired position of the guide stars to the actual measured position and the resulting three axis pointing errors. A small angle approximation was used for this derivation since stars seen by the video sensor are within a small angular distance of the telescope optical axis and a more sophisticated derivation using direction cosines or quaternions would result in equations that require an excessive amount of computer time to solve.

With the basic equations derived in appendix $A$, several methods were considered for deriving the final or best estimates of the three axis pointing errors. One approach considered was to use all the star data with a leastmean-squares approach assuming some error statistics associated with each star position measurement based on position in the CCD array and star magnitude. This method was compared to simply selecting star pairs, and solving the appropriate algebraic equations for the pointing errors. The least mean square approach resulted in more complex equations and, further, offered little benefit unless there was a great difference in the error statistics associated with the star measurements.

Consequently the approach taken for the multi-star processing is to select star pairs and the appropriate algebraic equations and then determine the three axis pointing errors. The resulting pointing errors for each star pair are then averaged together to obtain a best estimate.

Table 2 contains a summary of the algebraic equations for the pointing errors derived in appendix A. For each star pair there are two independent equations for roll and three independent equations for pitch and yaw. Assuming 
identically distributed, independent statistics for the measurements of star position, the variances of the attitude error equations are also included in table 2. Each of the algebraic equations has singularities as well and a selection of the appropriate equation must therefore be based on the relative location of the two stars. Table 3 summarizes the selection process. The only restriction occurs when two stars are in the same quadrant and the singularities in the equations and resultant amplification of error indicates that this case should be rejected as a valid star pair. of course there is an added restriction for the selection of star pairs to calculate the roll pointing error. Due to the geometry of using stars relatively close to the telescope line of sight, the roll pointing error is much larger than that for pitch and yaw and star pairs for roll should be selected as far from the origin within the field of view as possible.

The ultimate performance of the multi-star processing depends, of course, on the performance of the video sensor and the number of star pairs used, Performance of the CCD sensor will include two effects: accuracy and quantization of the interpolation calculations, and noise associated with random fluctuations of the levels in the individual CCD we1ls. Design of the CCD sensor ensures that the quantization effect in the interpolation calculations will be small and current test data shows that the interpolation calculations accuracy is approximately \pm 10 percent of the CCD well size. Noise associated with the CCD sensor star position measurements is also small compared to the interpolation accuracy and will not be considered in this section; however, it is important for design of the gyro filter.

- Since the interpolation error is the primary error source from the CCD sensor, a measure of the accuracy (neglecting misalignments) of the VIP system can be closely approximated as follows. If the interpolation error is assumed to be uniformly distributed then

$$
E\left\{\Delta x_{i}^{2} \text { or } \Delta y_{i}^{2}\right\}=\frac{a^{2}}{3} \triangleq \sigma^{2}
$$

where $a$ is ten percent of the CCD well dimension in arc sec. If three guide star pairs, allowing use of equations (A5a) from table 2, are assumed,

$$
\frac{x_{o i}^{2}+x_{o j}^{2}}{\left(x_{o i}-x_{o j}\right)^{2}} \text { and } \frac{y_{o i}^{2}+y_{o j}{ }^{2}}{\left(y_{o i}-y_{o j}\right)^{2}} \leq 1
$$

and

$$
E\left\{\theta_{P_{i}}^{2}\right\}=E\left\{\theta_{Y_{i}}^{2}\right\}=\sigma^{2}
$$

Since

$$
E\left\{\hat{\theta}_{P}^{2}\right\}=\frac{1}{N^{2}}\left[\sum_{i=1}^{N} E\left\{\theta_{P_{i}}{ }^{2}\right\}\right]
$$


then

$$
\mathrm{E}\left(\hat{\theta}_{\mathrm{p}}{ }^{2}\right)=\mathrm{E}\left(\hat{\theta}_{i}{ }^{2}\right) \leq \frac{\sigma^{2}}{3}
$$

Similarly, for roll, if three guide star pairs with the stars at least $1^{\circ}$ from the center, are assumed,

$$
\frac{1}{\left(y_{o i}-y_{o j}\right)^{2}} \text { and } \frac{1}{\left(x_{o i}-x_{o j}\right)^{2}} \leq \frac{180}{\pi}
$$

and

$$
\mathrm{E}\left(\theta_{\mathrm{R}}^{2}\right) \leq \frac{180 \times \dot{2} \times \sigma^{2}}{\pi}
$$

and

$$
E\left(\hat{\theta}_{R}^{2}\right) \leq \frac{180 \times 2 \times \sigma^{2}}{3 \pi}
$$

Table 4 summarizes the $1 \sigma$ null pitch and yaw pointing accuracies for both the $190 \times 244$ and $400 \times 400 \mathrm{CCD}$ array sizes. The poorer accuracy for roll is clearly shown and is a result of the geometry as explained earlier. While the roll numbers appear large, it must be remembered that this is accuracy and is not a measure of the roll stability due to quantization effects in the CCD sensor. The roll quantization would be much smaller. Table 4 clearly shows the effects and benefits of the multi-star processing.

\section{VIP HYBRID SIMULATION}

Operation of the complete VIP System electronics has been thoroughly tested utilizing a hybrid computer to simulate the functions of the CCD star tracker, the telescope dynamics, and the gyro-controlled stabilization system. Figure 11 is a block diagram representing the interrelationship of the major components of the simulation.

An HP-2114 digital computer is employed to generate the star field, perform the logic functions required to select stars that would be detected by the CCD star tracker, and format and output the data in a manner identical to the Intel 8080 microprocessor in the star tracker electronics. The telescope control system and dynamics are programmed on an EAI TR-48 analog computer. The body pitch, yaw, and roll rates are sampled directly from the analog computer by the digital computer which uses them to determine the apparent-motion of the star field. Simulated gyro outputs from the analog computer are compared to the computed pitch, yaw, and roll error angles calculated by VIP from the star tracker data. The results of this comparison is used to drive an update filter which applies the appropriate signals to the gyro torquers. Thus, the 2114 and $T R-48$ are interconnected and each has two-way communication with the VIP electronics package as well. 
The following section provides a brief description of the CCD star tracker simulation on the HP-2114 computer. Figure 12 illustrates an inertially-fixed coordinate system whose origin is at the telescope center of mass. Stars are located on an imaginary celestial sphere of unity radius centered about this coordinate system. The right ascension and declination for any star may be converted to the corresponding position vector by use of equations (16) below:

$$
\left.\begin{array}{l}
x_{S}=\cos \theta \sin \phi \\
y_{S}=\sin \theta \\
z_{S}=\cos \theta \cos \phi
\end{array}\right\}
$$

The right ascension, declination, and magnitude of up to 30 stars are stored in the HP-2114 memory. The field has been limited to $16^{\circ}$ square and several test patterns as well as constellations taken from star charts have been programmed. Figure 13 shows a typical pattern.

The location of a star in the body-fixed coordinate system of the telescope-star tracker can be calculated using the direction cosine matrix which relates the body and inertial coordinate systems. The elements of the direction cosine matrix are updated by the HP 2114 after each sample of the analog states representing the actual (as compared with the measured) body rates. Thus

$$
\underline{x}_{B}=\underline{x}_{S} .
$$

Since the star tracker is only concerned with the $x$ and $y$ coordinates, the computer actually calculates

$$
\left[\begin{array}{c}
x_{B} \\
y_{B}
\end{array}\right]=\left[\begin{array}{lll}
C_{11} & C_{12} & C_{13} \\
C_{21} & C_{22} & C_{23}
\end{array}\right]\left[\begin{array}{c}
x_{S} \\
y_{S} \\
z_{S}
\end{array}\right]
$$

The star tracker field of view has fixed angular dimensions that may be translated into the equivalent $x_{F}$, $y_{F}$ from equations (16). Program logic tests first each $x_{B}$, followed by the $y_{B}$ 's of the stars that remain. So that if both

$$
\left.\begin{array}{l}
-\mathrm{x}_{\mathrm{F}} \leq \mathrm{x}_{\mathrm{B}_{i}} \leq \mathrm{x}_{\mathrm{F}} \\
-\mathrm{y}_{\mathrm{F}} \leq \mathrm{y}_{\mathrm{B}_{i}} \leq \mathrm{y}_{\mathrm{F}}
\end{array}\right\}
$$

the coordinates of that star are saved. 
The integration time and threshold are selectable functions from the VIP control panel. During each data cycle, the status of these functions is sampled and the following algorithm is used to determine the $M_{v}$ of the dimmest star capable of being detected by the tracker:

$$
M_{v} \leq(6.75-j)+0.25 i
$$

where $j$ is the threshold level and $i$ is the integration-time switch position. Thus, stars are eliminated from the tracker field of view if they are dimmer than the maximum $\mathrm{M}_{\mathrm{V}}$ calculated in equation (20).

Two additional functions are required for the star tracker simulation; data formatting and time delay. These are both performed at the end of the computation cycle. Formatting is necessary because a) the coordinate system used by the star tracker has its origin in the lower right corner instead of the center, b) the data must be delivered to the VIP interface in the correct sequence and timing, and $c$ ) the data must be scaled from the $x_{B}, y_{B}, M_{v}$ values used for calculation to the values the star tracker would actualiy output. The scaling simulates both the interpolation and quantization inherent in the CCD. A time delay in the real tracker results from the period of integration and the computation necessary to do interpolation. The HP-2114 logic automatically adjusts the time delay to the appropriate value according to the integration time requested by the VIP control panel switch. Figure 14 is a flow chart for the HP-2114 program indicating the major steps required for the simulation. Figure 15 is a photograph of the experimental set-up showing the major components.

The results of tests using the hybrid simulation are shown in figures 16(a) and (b). The relatively slow response of the filter and the somewhat under-damped nature of the commanded attitude response were predicted by the $\mathrm{Z}$-plane analysis discussed in the previous section. The performance is more than adequate, however, and with the addition of the commanded star field display option, described in an earlier section, the operator may position the optical axis without having to contend with the system dynamics.

\section{CONCLUSTONS}

Design and performance details of a first generation system and an advanced Video Inertial Pointing system capable of providing three-axis pointing error information with a single video sensor have been presented. The results indicate that the concept is a viable one. The use of a multistar processing to generate three-axis error signals has also been shown to substantially improve star-position measurement statistics when a simple averaging algorithm is included. An analysis of the problem of using delayed, quantized star tracker information to update the output of the gyro stabilization loop revealed the importance of careful modeling of the discrete portion of the system. A hybrid simulation in combination with work done at the Jet Propulsion Laboratory on the VIP CCD video sensor (see 2) has 
demonstrated the sucessful integration of state-of-the-art devices such as microprocessors and a solid-state video star tracker in an operating system. It is anticipated that the VIP system concept will be part of the Shuttle Infrared Telescope Facility as well as other systems used to point instruments on the Space Shuttle. 
Consider the sma11-angle approximation to the direction cosine matrix which transforms inertial to body-fixed coordinates through orthogonal pitch, yaw, and roll angles:

$$
\left[\begin{array}{ccc}
1 & \theta_{\mathrm{R}} & -\theta_{\mathrm{y}} \\
-\theta_{\mathrm{R}} & 1 & { }_{\mathrm{p}} \\
\theta_{\mathrm{y}} & -\theta_{\mathrm{p}} & 1
\end{array}\right]
$$

The position of a star in body coordinates after a small angle rotation is

$$
\left[\begin{array}{c}
x_{B} \\
y_{B} \\
z_{B}
\end{array}\right]=\left[\begin{array}{ccc}
1 & \theta_{R} & -\theta_{y} \\
-\theta_{R} & 1 & \theta_{p} \\
\theta_{y} & -\theta_{p} & 1
\end{array}\right]\left[\begin{array}{c}
x_{I} \\
y_{I} \\
z_{I}
\end{array}\right]
$$

Since we're interested in the $x_{B}, y_{B}$ components only, we may expand them as

$$
\left.\begin{array}{l}
x_{B}=x_{I}+\theta_{R} y_{I}-\theta_{Y} z_{I} \\
y_{B}=-\theta_{R} x_{I}+y_{I}+\theta_{P} z_{I}
\end{array}\right\}
$$

If $x_{I}, y_{I}$ is considered to be the initial location of the star, then the change in position, as seen by the star-tracker is

$$
\left.\begin{array}{l}
\Delta \mathrm{x} \triangleq \mathrm{x}_{\mathrm{B}}-\mathrm{x}_{\mathrm{I}} \\
\Delta \mathrm{y} \triangleq \mathrm{y}_{\mathrm{B}}-\mathrm{y}_{\mathrm{I}}
\end{array}\right\}
$$

Equations (A3) may be normalized by dividing through by $z_{I}$, since $z_{I} \gg x_{I}, y_{I}$ and the $z_{I}^{\prime}$ 's are essentially equal for all stars. Defining $k$ as the scaling factor for the normalization and the conversion of the image through the electro-optical system of the star tracker, equations (A3) may be rewritten as:

$$
\left.\begin{array}{l}
\mathrm{K} \Delta \mathrm{x}=\theta_{\mathrm{R}} \mathrm{Ky}_{\mathrm{O}}-\theta_{\mathrm{Y}} \\
\mathrm{K} \Delta \mathrm{y}=-\theta_{\mathrm{R}} \mathrm{Kx_{0 }}+\theta_{\mathrm{P}}
\end{array}\right\}
$$


Thus, for two stars, using the $\Delta x$ equation

$$
\left.\begin{array}{l}
\theta_{R}=\frac{\Delta x_{i}-\Delta x_{j}}{\left(y_{o i}-y_{o j}\right)} \\
\theta_{y}=\frac{K\left(\Delta x_{i} y_{o j}-\Delta x_{j} y_{o i}\right)}{\left(y_{o i}-y_{o j}\right)}
\end{array}\right\}
$$

and for the $\Delta y$ equation

$$
\begin{aligned}
& \theta_{R}=\frac{\Delta y_{i}-\Delta y_{j}}{\left(x_{o j}-x_{o i}^{\prime}\right)} \\
& \theta_{P}=\frac{k\left(\Delta y_{i} x_{o j}-\Delta y_{j} x_{o i}\right)}{\left(x_{o j}-x_{o i}\right)}
\end{aligned}
$$

An additional set of equations for $\theta_{\mathrm{P}}$ and $\theta_{\mathrm{Y}}$ may be obtained by using the $\theta_{\mathrm{R}}$ derived from the $\Delta \mathrm{y}$ portion of (A5) in the $\Delta \mathrm{x}$ equation and vice versa. They are

$$
\left.\begin{array}{rl}
\theta_{Y} & =\frac{k\left[y_{o i}\left(\Delta y_{i}-\Delta y_{j}\right)+\Delta x_{i}\left(x_{o i}-x_{o j}\right)\right]}{\left(x_{o j}-x_{o i}\right)} \\
. \theta_{P} & =\frac{k\left[x_{o i}\left(\Delta x_{i}-\Delta x_{j}\right)+\Delta y_{i}\left(y_{o i}-y_{o j}\right)\right]}{\left(y_{o i}-y_{o j}\right)}
\end{array}\right\}
$$

Using equations (A6) and (A7) for a star field of $n$ stars, we may improve our calculation of $\theta_{P, Y, R}$ by averaging the results. Thus

$$
\begin{aligned}
& \bar{\theta}_{P}=\frac{2 \mathrm{k}}{n(n-1)} \sum_{i=1}^{n}\left\{\sum_{j=i+1}^{n}\left[\frac{\left(\Delta y_{i j} x_{o j}-\Delta y_{j} x_{o i}\right)}{\left(x_{o j}-x_{o j}\right)}\right]\right\} \\
& \bar{\theta}_{y}=\frac{2 \mathrm{~K}}{\mathrm{n}(\mathrm{n}-1)} \sum_{i=1}^{\mathrm{n}}\left\{\sum_{j=i+1}^{\mathrm{n}}\left[\frac{\left(\Delta \mathrm{x}_{i} \mathrm{y}_{\mathrm{oj}}-\Delta \mathrm{x}_{j} \mathrm{y}_{\mathrm{oi}}\right)}{\left(\mathrm{y}_{\mathrm{o} i}-\mathrm{y}_{\mathrm{oj}}\right)}\right]\right\} \\
& \bar{\theta}_{R}=\frac{1}{n(n-1)}\left\{\sum_{i=1}^{n}\left[\sum_{j=1+i}^{n} \frac{\left(\Delta x_{i}-\Delta x_{j}\right)}{\left(y_{o i}-y_{o j}\right)}-\frac{\left(\Delta y_{i}-\Delta y_{j}\right)}{\left(x_{o i}-x_{o j}\right)}\right]\right\}
\end{aligned}
$$


We may also wish to utilize some form of weighted averaging based on, for example, the relative brightness of the star, the separation of a star pair, or the distance of the star from the telescope line of sight (for $\bar{\theta}_{\mathrm{R}}$ calculations). Given some weighting factor $W_{i j}=f\left(x_{i}, y_{i}, x_{o i}, y_{o i}, x_{j}, y_{j}, x_{o j}\right.$,
$\left.y_{o j}, M_{v_{i}}, M_{v_{j}}\right)$ then equations (A9) become

$$
\bar{\theta}_{P}=\frac{\mathrm{k}}{\sum_{i=1}^{\mathrm{n}} \sum_{j=i+1}^{\mathrm{n}} \sum_{\mathrm{P}_{i j}}} \sum_{i=1}^{\mathrm{n}}\left\{\sum_{j=i+1}^{\mathrm{n}}\left[\frac{\left(\Delta \mathrm{y}_{i} \mathrm{x}_{\mathrm{oj}}-\Delta \mathrm{y}_{j} \mathrm{x}_{\mathrm{oi}}\right) \mathrm{w}_{P i j}}{\left(\mathrm{x}_{\mathrm{oj}}-\mathrm{x}_{\mathrm{oi}}\right)}\right]\right\}
$$

$$
\begin{aligned}
& \vec{\theta}_{y}=\frac{n}{\sum_{i=1}^{n} \sum_{j=i+1}^{n} W_{y i j}} \sum_{i=1}^{n}\left\{\sum_{j=i+1}^{n}\left[\frac{\left(\Delta x_{i} y_{o j}-\Delta x_{j} y_{o i}\right) w_{Y i j}}{\left(y_{o i}-y_{o j}\right)}\right]\right\} \\
& \bar{\theta}_{R}=\frac{1}{2 \sum_{i=1}^{n} \sum_{j=i+1}^{n} W_{R 1 i j}}\left\{\sum_{i=1}^{n}\left[\sum_{j=i+1}^{n} \frac{\left(\Delta x_{i}-\Delta x_{j}\right) W_{R 1 i j}}{\left(y_{o i}-y_{o j}\right)}\right]\right\}
\end{aligned}
$$

$$
-\frac{1}{2 \sum_{i=1}^{n} \sum_{j=i+1}^{n} w_{R 2 i j}}\left\{\sum_{i=1}^{n}\left[\sum_{j=i+1}^{n} \frac{\left(\Delta y_{i}-\Delta y_{j}\right) w_{R 2 i j}}{\left(x_{o i}-x_{o j}\right)}\right]\right\}
$$




\section{REFERENCES}

1. McCarthy, S. G.; Young, L. S.; and Witteborn, F. C.: A Large Cooled Infrared Telescope Facility for Spacelab. 21st Annual Meeting of the American Astronautical Society, Denver, Co, Aug. 24-26, 1975.

2. Saloman, P. M.; and Goss, W. C.: A Microprocessor-Controlled CCD Star Tracker. 14th Aerospace Sciences Meeting, AIAA, Washington, D. C., Jan. 28-30, 1976.

3. Swift, C. D.; Witteborn, F. C.; and Shipley, A.; et al. (eds.): Proceedings of the Telescope Systems for Balloon-Borne Research Symposium. NASA TM X-62,379, 1974.

4. Farrenkopf, R. L.: Generalized Results' for Precision Attitude Reference Systems Using Gyros. AIAA Mechanics and Control of Flight Conference, Anaheim, CA, Aug. 5-9, 1974.

5. Rauch, H. E.: Correlation of Line-of-Sight Errors - An Analytic Error Analysis. LMSC-D267918, Rev. I, Lockheed-Palo A1to Research Laboratory, Palo Alto, CA, June 1972.

6. Potter, J. E.; and Vander Velde, W. E.: Optimum Mixing of Gyroscope and Star Tracker Data. Journal of Spacecraft and Rockets, vo1. 5, no. 5, 1968.

7. Truxall, John G.: Automatic Feedback Control System Synthesis, McGrawHill Book Co., Inc., New York, 1955, pp. 548-550.

8. Powell, J. D.; and Throckmorton, A. J.: A Study of Filter Mechanizations for the Video Inertial Pointing System Microprocessor. Final Report, Guidance and Control Laboratory, Dept. of Aeronautics and Astronautics, Stanford University, Stanford, CA, Oct. 1975. 
TABLE 1.- STAR TRACKER DYNAMIC RANGE FOR SEVEN THRESHOLD LEVELS AND SEVEN INTEGRATION TIMES

\begin{tabular}{|c|c|c|c|c|c|c|c|}
\hline $\begin{array}{l}\text { Thresh } \\
\text { T Ievel } \\
\text { sec }\end{array}$ & $\mathrm{TH} 1$ & $\mathrm{TH} 2$ & $\mathrm{TH} 3$ & $\mathrm{TH} 4$ & $\mathrm{TH} 5$ & $\mathrm{TH} 6$ & TH 7 \\
\hline$T_{1}$ & $Q>0.40$ & $Q>1.0$ & $Q>2.5$ & $Q>6.3$ & $Q>16$ & $Q>40$ & $Q>100$ \\
\hline 0.250 & $2<\mathrm{M}_{\dot{\mathrm{v}}}<7$ & $1<\mathrm{M}_{\mathrm{v}}<6$ & $0<\mathrm{M}_{\mathrm{v}}<5$ & $-1<\mathrm{M}_{\mathrm{v}}<4$ & $-1<\mathrm{M}_{\mathrm{v}}<3$ & $-1<M_{\mathrm{v}}<2$ & $-1<M_{v}<1$ \\
\hline \multicolumn{8}{|l|}{$\mathrm{T}_{2}$} \\
\hline .315 & $2.25,7.25$ & $1.25,6.25$ & $-0.25,5.25$ & $-0.75,4.25$ & $-1,3.25$ & $-1,2.25$ & $-1,1.25$ \\
\hline \multicolumn{8}{|l|}{$\mathrm{T}_{3}$} \\
\hline .396 & $2.5,7.5$ & $1.5,6.5$ & $0.5,5.5$ & $-0.5,4.5$ & $-1,3.5$ & $=1,2.5$ & $-1,1.5$ \\
\hline \multicolumn{8}{|l|}{$\mathrm{T}_{4}$} \\
\hline .499 & $2.75,7.75$ & $1.75,6.75$ & $0.75,5.75$ & $-0.25,4.75$ & $-1,3.75$ & $-1,2.75$ & $-1,1.75$ \\
\hline \multicolumn{8}{|l|}{$\mathrm{T}_{5}$} \\
\hline .628 & $3.0,8.0$ & $2.0,7.0$ & $1,6.0$ & $0,5.0$ & $-1,4.0$ & $-1,3.0$ & $-1,2.0$ \\
\hline \multicolumn{8}{|l|}{$\mathrm{T}_{6}$} \\
\hline .791 & $3.25,9.25$ & $2.25,7.25$ & $1.25,6.25$ & $0.25,5.25$ & $-0.75,4.25$ & $-1,3.25$ & $-1,2.25$ \\
\hline \multicolumn{8}{|l|}{$\mathrm{T}_{7}$} \\
\hline .995 & $3.5,8.5$ & $2.5,7.5$ & $1.5,6.5$ & $0.5,5.5$ & $-0.5,4.5$ & $-1,3.5$ & $-1,2.5$ \\
\hline
\end{tabular}


TABLE 2.- EXPECTED VALUE OF ATTITUDE ERROR FOR EACH OF THE FIVE TYPES OF ATTITUDE ERROR EQUATIONS

\begin{tabular}{|c|c|c|c|c|}
\hline & Attitude error equation & E\{Attitude error $\left.{ }^{2}\right\} *$ & & Singularities \\
\hline \multirow{3}{*}{$\theta_{p}$} & $\frac{k\left(x_{o j} \Delta y_{i}-x_{o i} \Delta y_{j}\right)}{\left(x_{o j}-x_{o i}\right)}$ & $\frac{\sigma^{2}\left(x_{o i}^{2}+x_{o j}^{2}\right) k^{2}}{\left(x_{o j}-x_{o i}\right)^{2}}$ & $x_{o i}=x_{o j}$ & $\begin{array}{l}\text { Amplification of errors when } \\
x_{o i} \text { and } x_{o j} \text { are of same sign. }\end{array}$ \\
\hline & $\frac{k\left[x_{o i}\left(\Delta x_{i}-\Delta x_{j}\right)+\Delta y_{i}\left(y_{o i}-y_{o j}\right)\right]}{\left(y_{o i}-y_{o j}\right)}$ & $\frac{k^{2} \sigma^{2}\left[\left(y_{o j}^{2}+y_{o j}^{2}\right)+2 x_{o i}^{2}\right]}{\left(y_{o i}-y_{o j}\right)^{2}}$ & $y_{o i}=y_{o j}$ & $\begin{array}{l}\text { Amplification of errors when } \\
y_{o i} \text { and } y_{o j} \text {, are of same sign. }\end{array}$ \\
\hline & $\frac{k\left[x_{o j}\left(\Delta x_{j}-\Delta x_{i}\right)+\Delta y_{j}\left(y_{o j}-y_{o j}\right)\right]}{\left(y_{o j}-y_{o i}\right)}$ & $\frac{\mathrm{k}^{2} \sigma^{2}\left[\left(\mathrm{y}_{\mathrm{oj}}^{2}+\mathrm{y}_{\mathrm{oi}}^{2}\right)+2 \mathrm{x}_{\mathrm{oj}}^{2}\right]}{\left(\mathrm{y}_{\mathrm{oj}}-\mathrm{y}_{\mathrm{oi}}\right)^{2}}$ & $y_{o i}=y_{o j}$ & $\begin{array}{l}\text { Amplification of errors when } \\
y_{o i} \text { and } y_{o j} \text { are of same sign. }\end{array}$ \\
\hline \multirow{3}{*}{$\theta_{y}$} & $\frac{K\left(i x_{i} y_{o j}-\Delta x_{j} y_{o j}\right)}{\left(y_{o i}-y_{o j}\right)}$ & $\frac{\sigma^{2} \mathrm{~K}^{2}\left(\mathrm{y}_{o i}^{2}+y_{o j}^{2}\right)}{\left(y_{o i}-y_{o j}\right)^{2}}$ & $y_{o i}=y_{o j}$ & $\begin{array}{l}\text { Amplification of errors when } \\
y_{o i} \text { and } y_{o j} \text { are of same sign. }\end{array}$ \\
\hline & $\frac{k\left[y_{o i}\left(\Delta y_{i}-\Delta y_{j}\right)+\Delta x_{i}\left(x_{o i}-x_{o j}\right)\right]}{\left(x_{o j}-x_{o i}\right)}$ & $\frac{x^{2} \sigma^{2}\left[\left(x_{o i}^{2}+x_{o j}^{2}\right)+2 y_{o i}^{2}\right]}{\left(x_{o i}-x_{o j}\right)^{2}}$ & $x_{o i}=x_{o j}$ & $\begin{array}{l}\text { Amplification of errors when } \\
\mathrm{x}_{o j} \text { and } \mathrm{x}_{\mathrm{oj}} \text { are of same sign. }\end{array}$ \\
\hline & $\frac{k\left[y_{o j}\left(\Delta y_{j}-\Delta y_{i}\right)+\Delta x_{j}\left(x_{o j}-x_{o i}\right)\right]}{\left(x_{o i}-x_{o j}\right)}$ & $\frac{k^{2} o^{2}\left[\left(x_{o i}^{2}+x_{o j}^{2}\right)+2 y_{o j}^{2}\right]}{\left(x_{o j}-x_{o i}\right)^{2}}$ & $x_{o i}=x_{o j}$ & $\begin{array}{l}\text { Amplification of errors when } \\
x_{o i} \text { and } x_{o j} \text { are of same sign. }\end{array}$ \\
\hline \multirow{2}{*}{$\theta_{\mathrm{R}}$} & $\frac{\Delta y_{i}-\Delta y_{j}}{\left(x_{o j}-x_{o i}\right)}$ & $\frac{2 \sigma^{2}}{\left(x_{o j}-x_{o i}\right)^{2}}$ & $x_{o i}=x_{o j}$ & $\begin{array}{l}\text { Best performance when } x_{0 i} \text { and } \\
x_{o j} \text { are of opposite sign. }\end{array}$ \\
\hline & $\frac{\Delta x_{i}-\Delta x_{j}}{\left(y_{o i}-y_{o j}\right)}$ & $\frac{2 \sigma^{2}}{\left(y_{o i}-y_{o j}\right)^{2}}$ & $y_{o i}=y_{o j}$ & $\begin{array}{l}\text { Best performance when } \mathrm{y}_{o i} \text { and } \\
\mathrm{y}_{o j} \text { are of opposite sign. }\end{array}$ \\
\hline
\end{tabular}

*Assumes that $\Delta \mathrm{x}_{i}$ and $\Delta \mathrm{y}_{i}$ are identically distributed independent random variables.. $\quad \mathrm{E}\left\{\Delta \mathrm{x}_{i}^{2}\right\}=\mathrm{E}\left\{\Delta \mathrm{y}_{i}^{2}\right\} \triangleq \sigma^{2}$ 
TABLE 3.- STAR-PAIR SELECTION CRITERIA

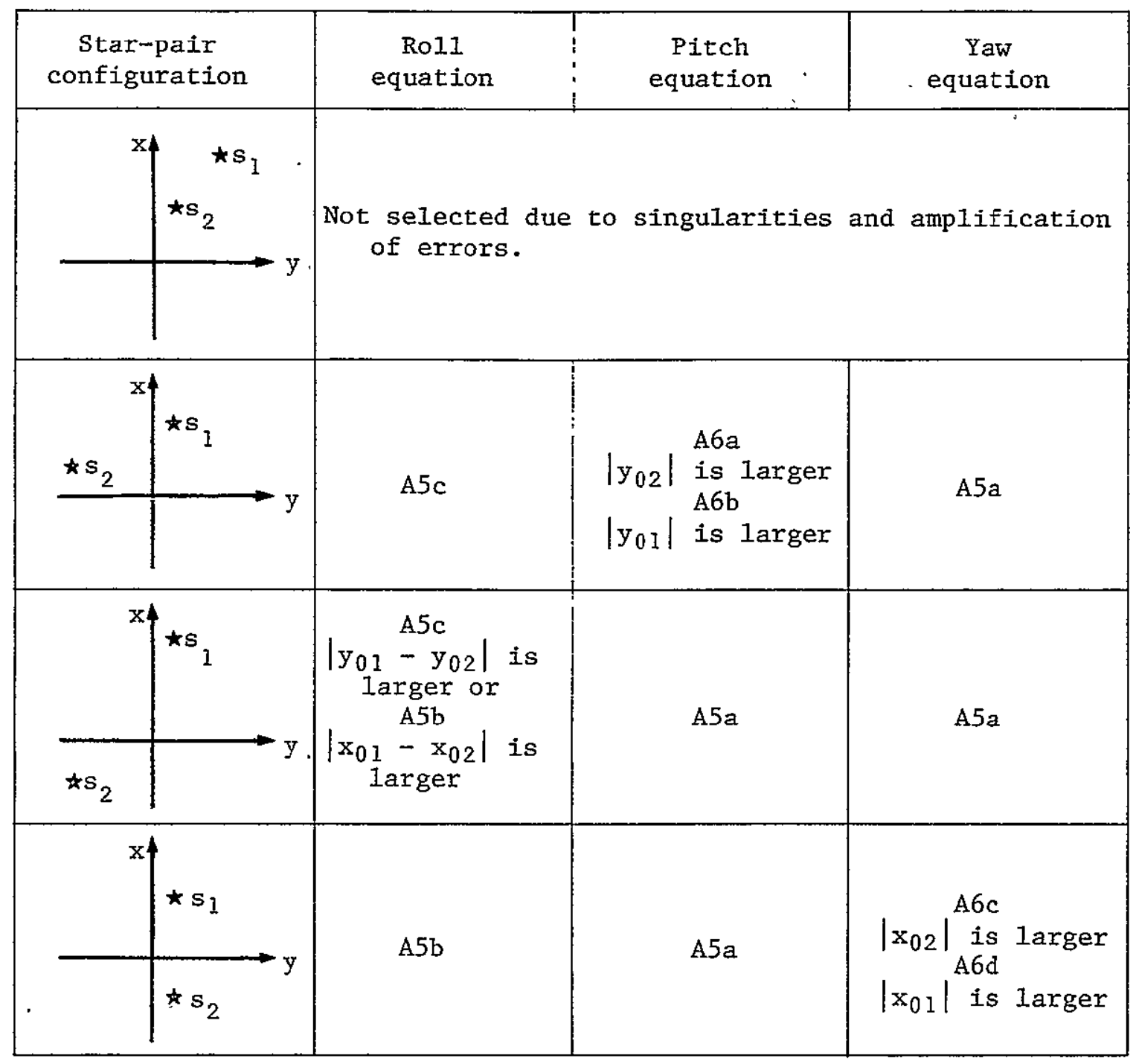


TABLE 4.- 1- $\sigma$ ROLL, PITCH, YAW POINTING ACCURACY (IN ARC SEC) WHILE TRACKING 4 STARS

\begin{tabular}{|c|c|c|c|c|c|}
\hline $\begin{array}{c}\text { Array size } \\
(\text { field of view) }\end{array}$ & $a$ & $\sigma_{\mathrm{r}}$ & $\sigma_{\mathrm{r}}$ & $\sigma_{\mathrm{pVE}}$ & $\sigma_{\mathrm{p}, \mathrm{y}}{ }_{\mathrm{AVE}}$ \\
\hline $\begin{array}{c}190 \times 244 \\
\left(1.9^{\circ} \times 2.5^{\circ}\right)\end{array}$ & 3.7 & $\leqslant 170$ & $\leqslant 70$ & $\leqslant 2.1$ & $\leqslant 0.9$ \\
$\begin{array}{c}400 \times 400 \\
\left(2^{\circ} \times 2^{\circ}\right)\end{array}$ & -1.8 & $\leqslant 84$ & $\leqslant 34$ & $\leqslant 1.0$ & $\leqslant 0.4$ \\
\hline
\end{tabular}




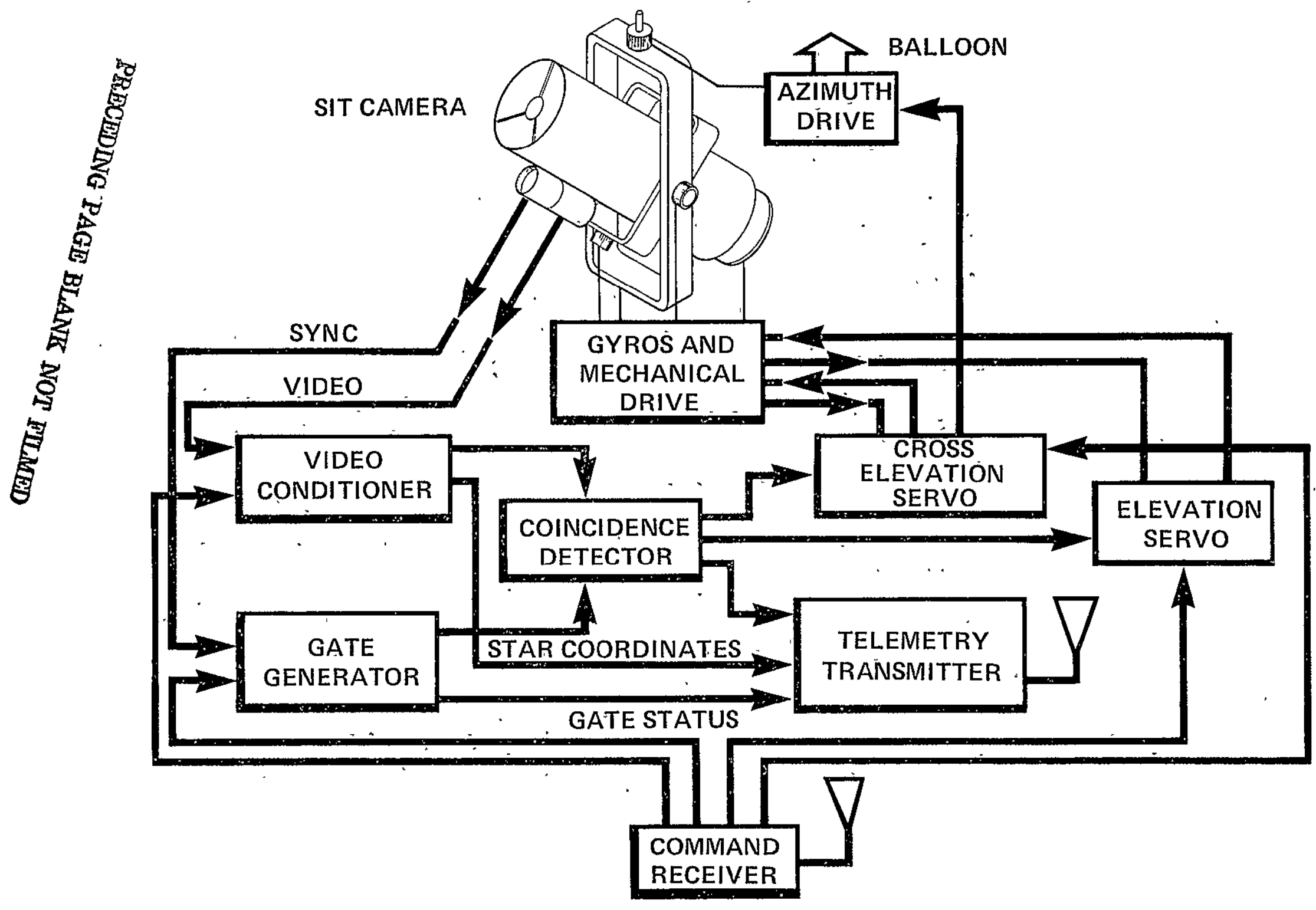

(a) Airborne portion.

Figure 1,- First generation VIP'system. 


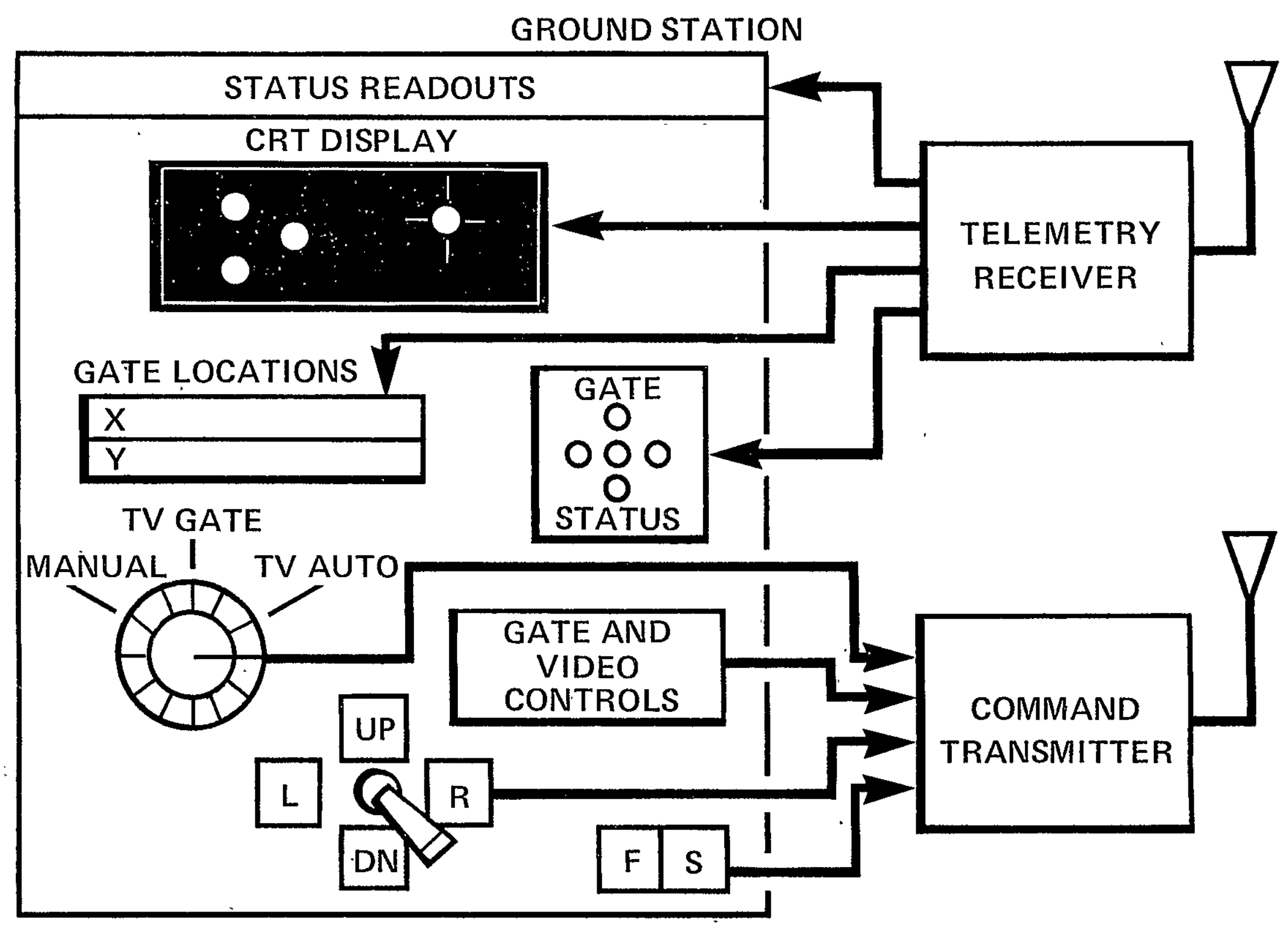

(b) Ground station.

Figure 1.- Concluded. 


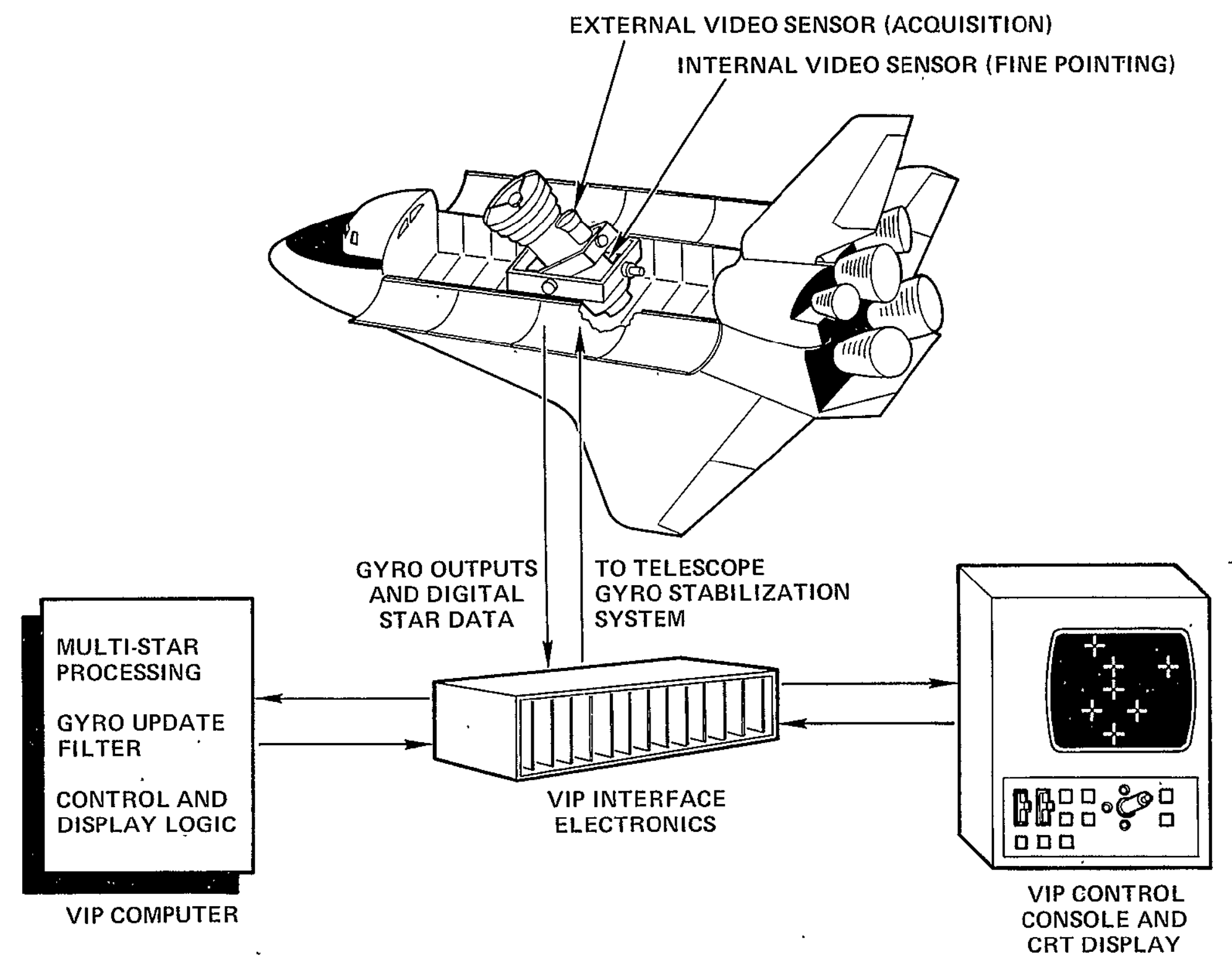

Figure 2.- Advanced VIP system. 


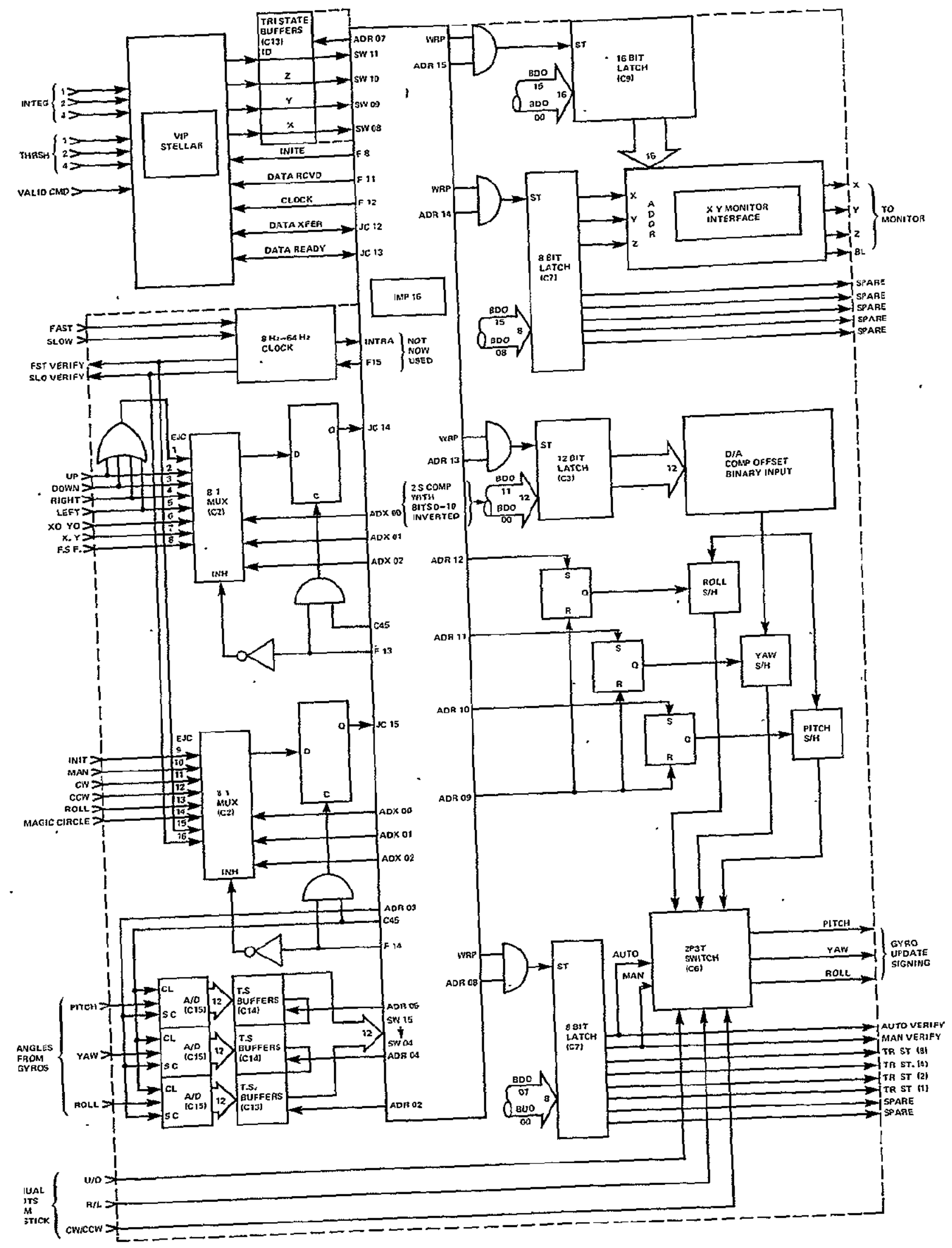

Figure 3.- VIP microprocessor and interface electronics block diagram. 


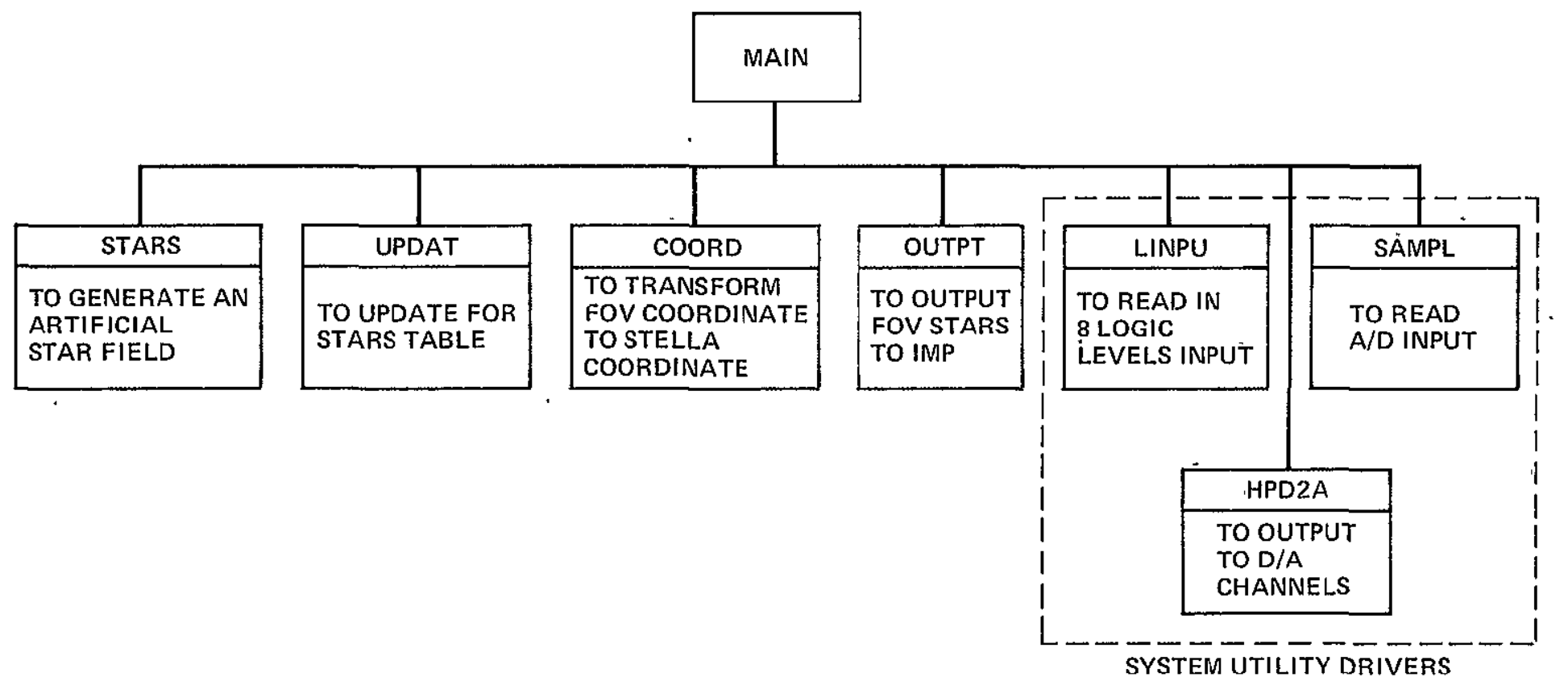

(a) HP-2114 star field/tracker simulation program structure.

Figure 4.- VIP microprocessor software organization and flow chart. 


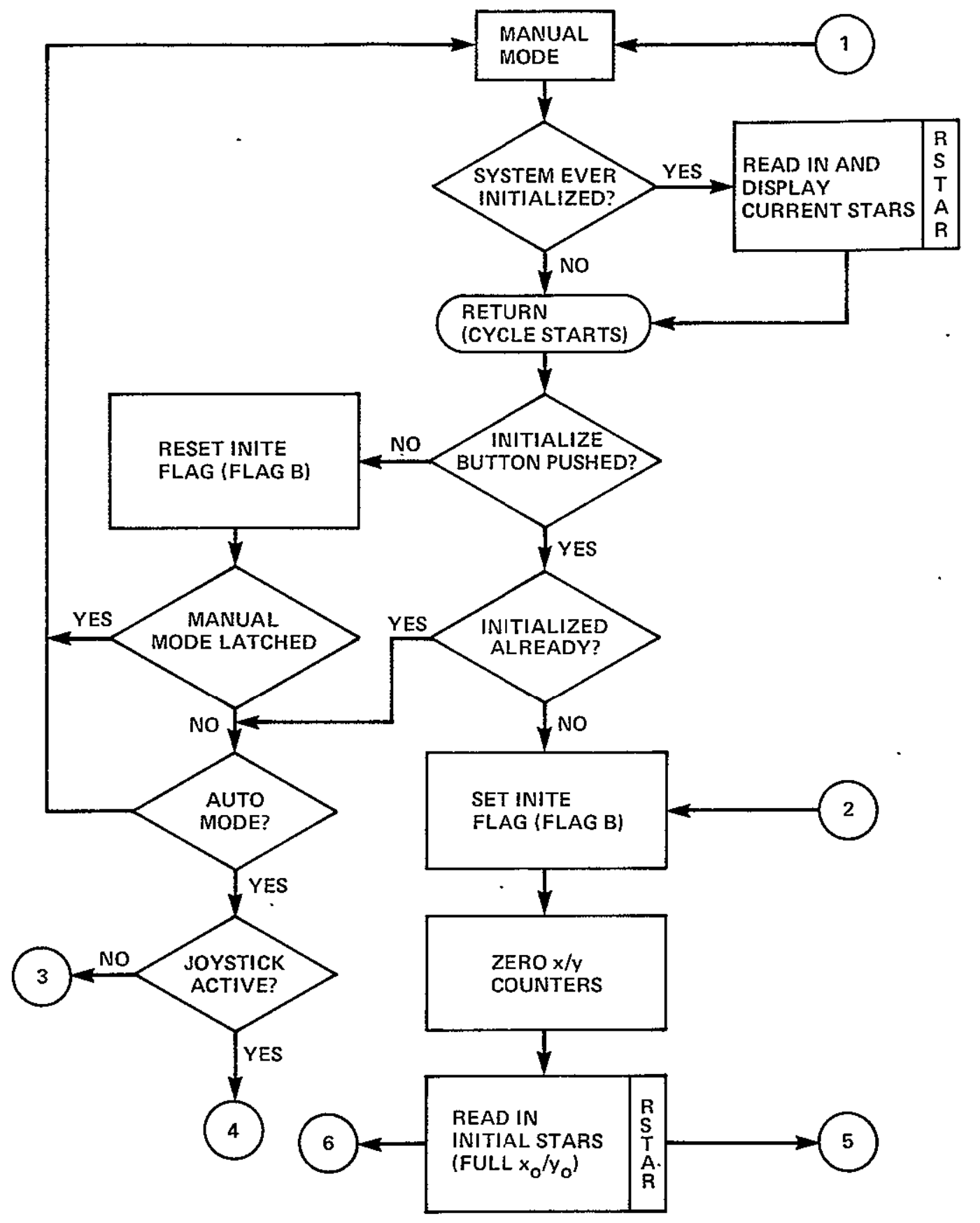

(b) VIP software flow chart.

Figure 4.- Continued. 


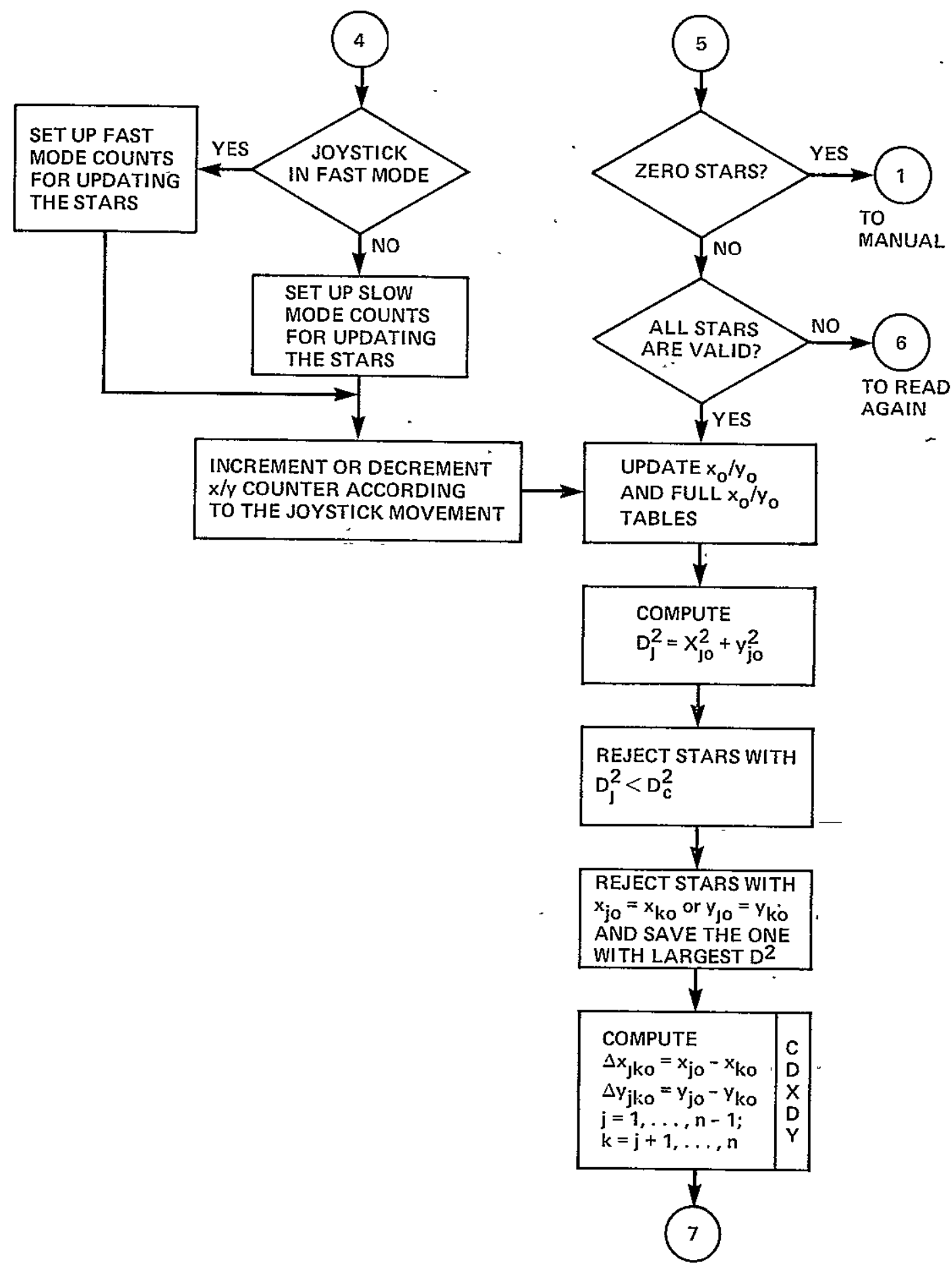

(c) VIP software flow chart.

Figure 4.- Continued. 


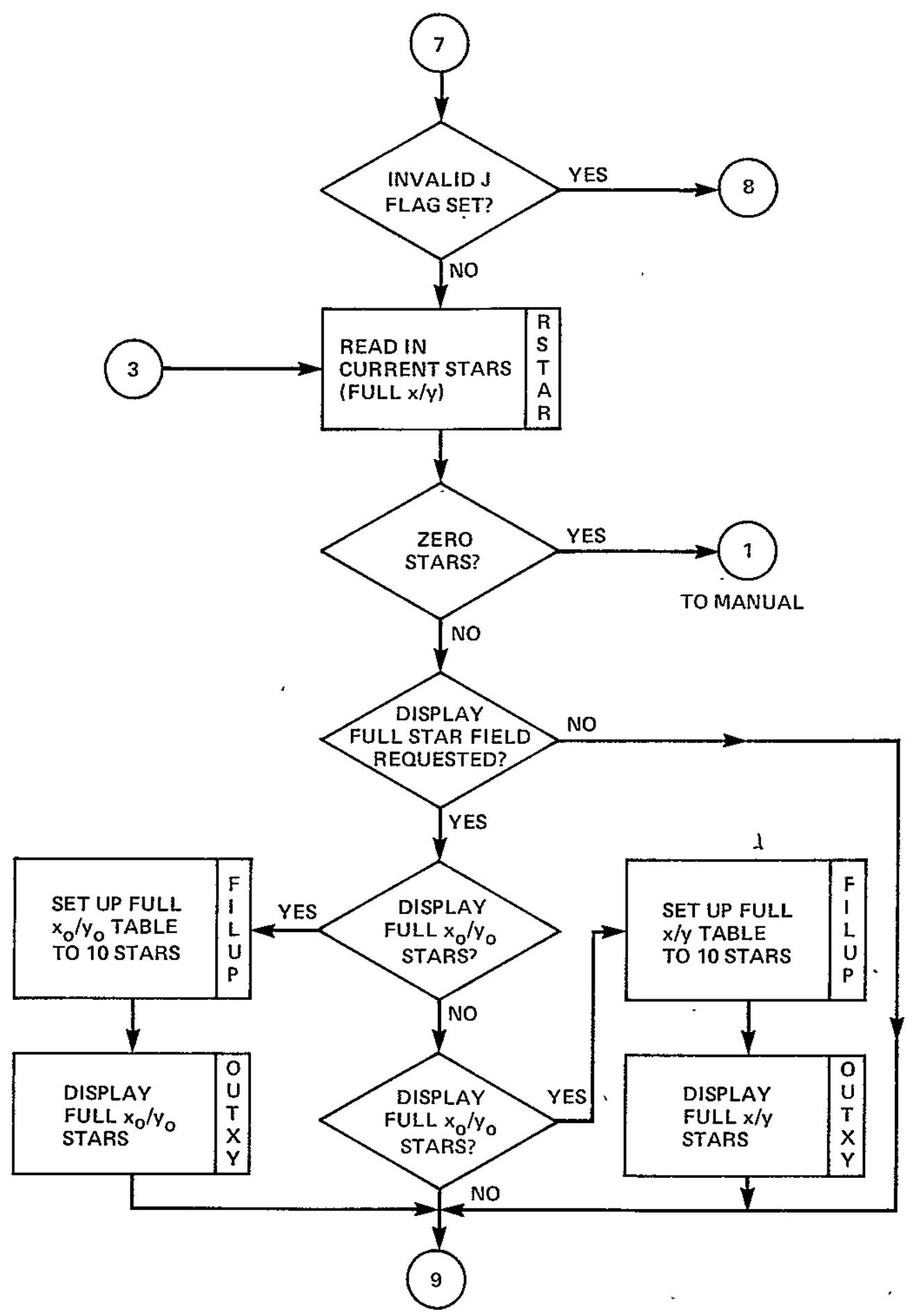

(d) VIP software flow chart.

Figure 4.- Continued. 


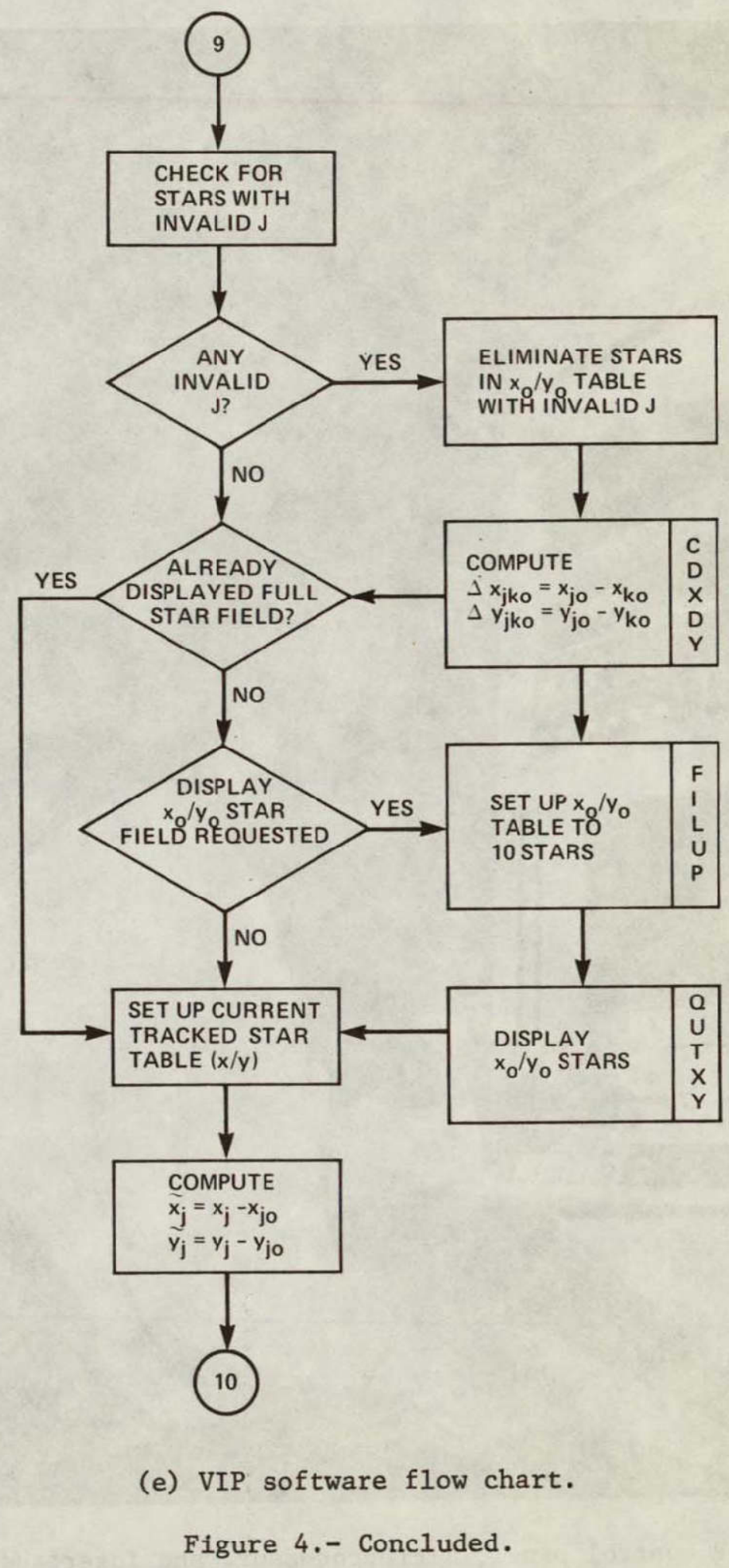




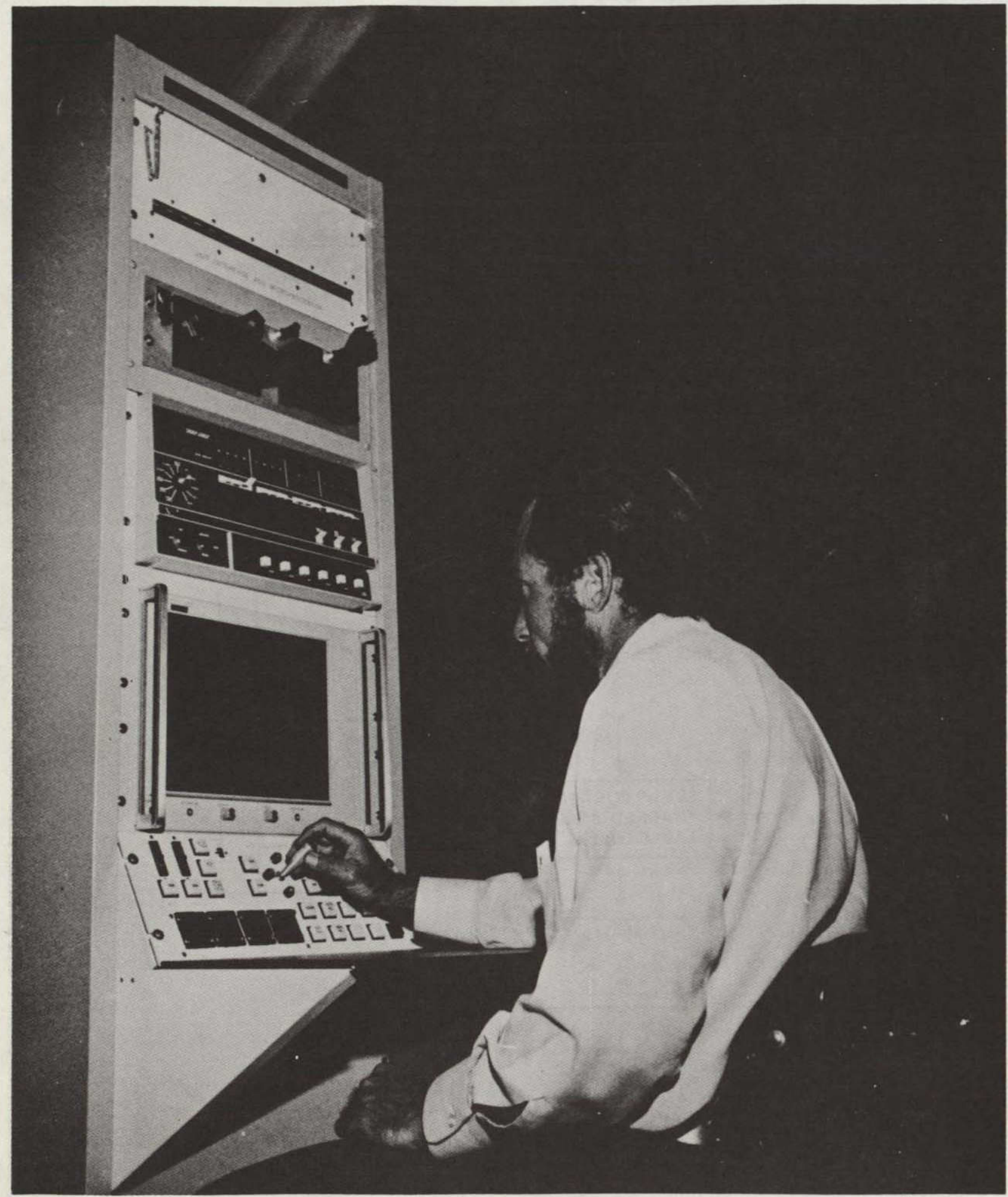

Figure 5.- VIP control panel, microprocessor, and interface electronics. 


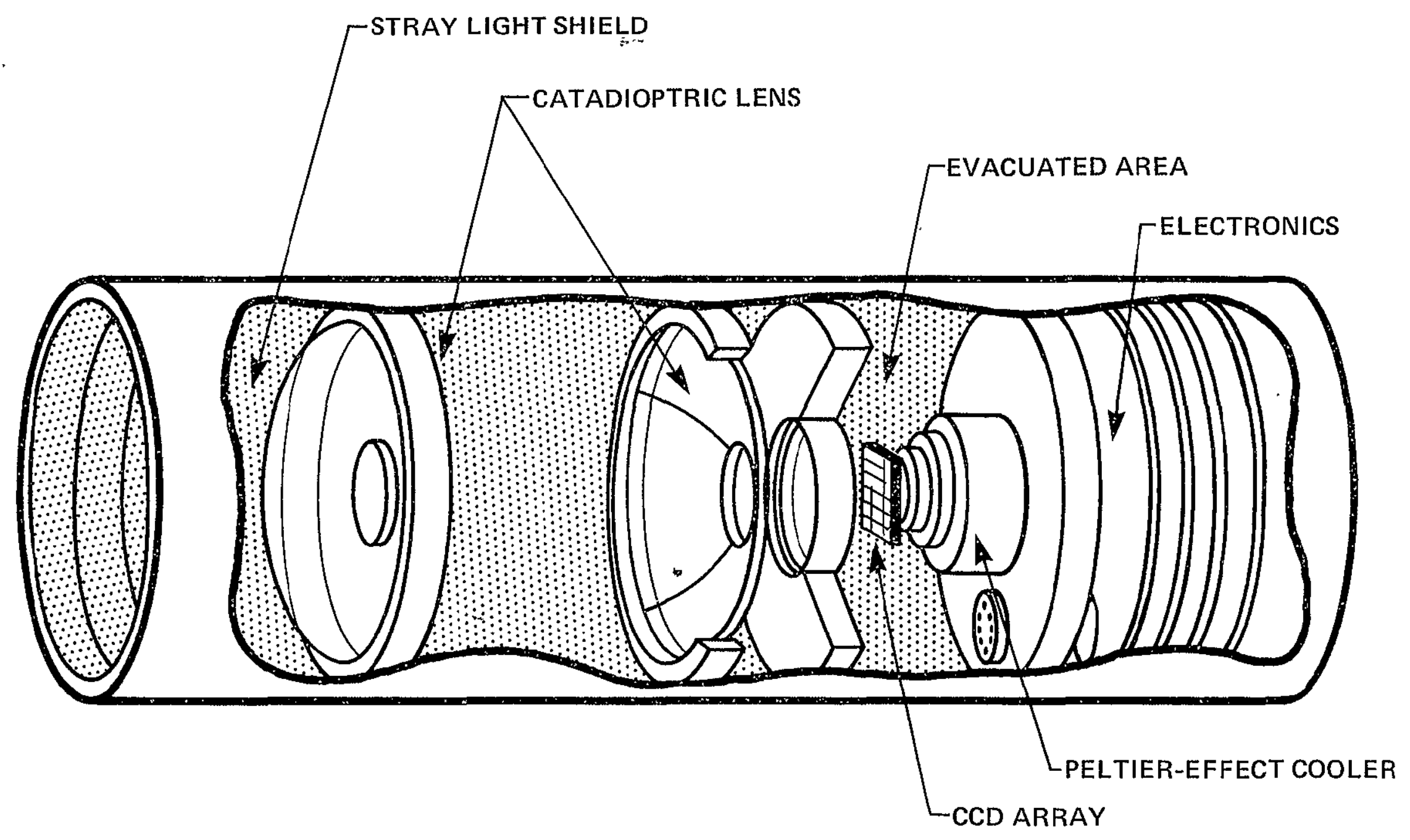

Figure 6.- CCD star tracker. 


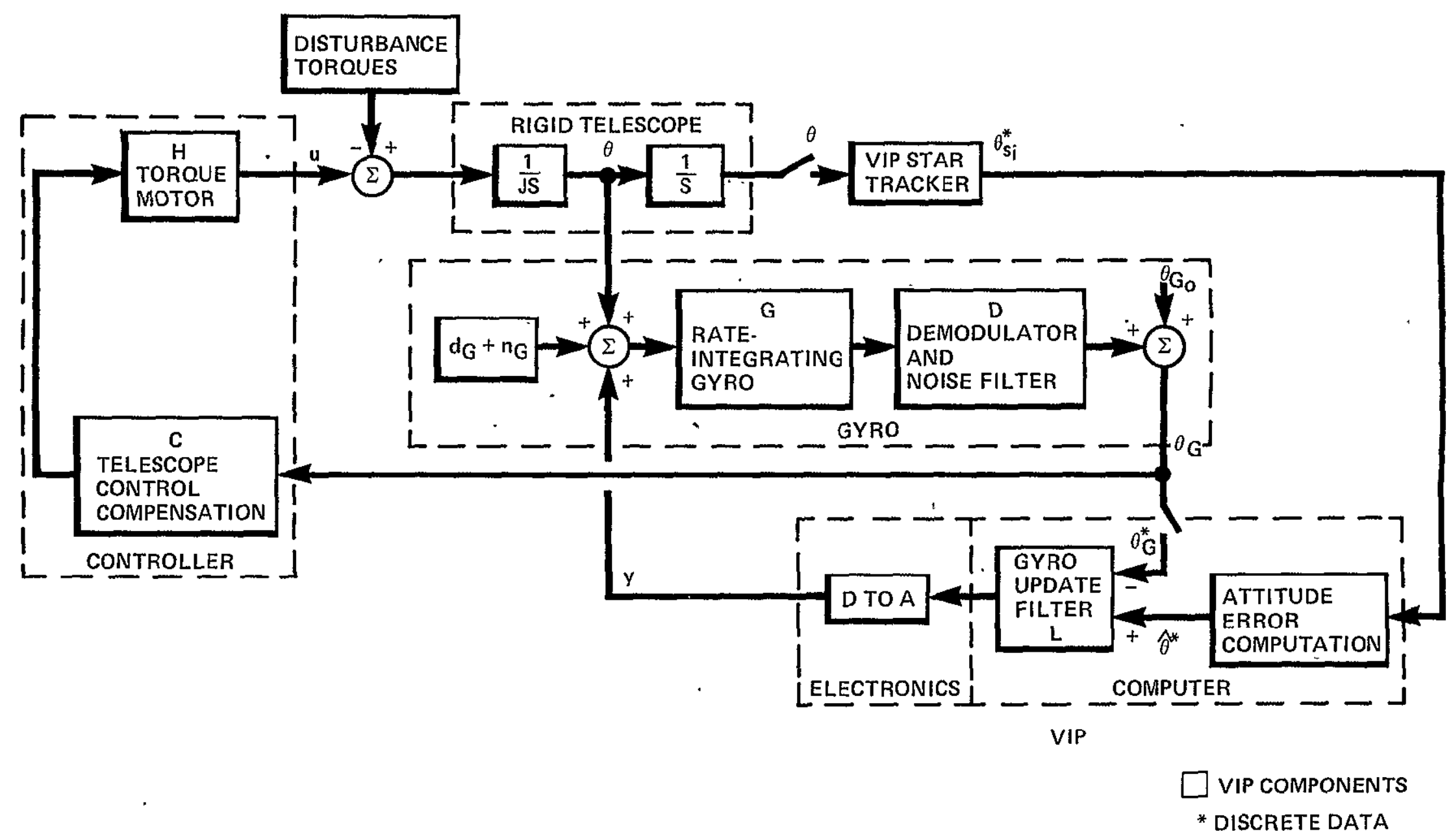

Figure 7.- Block diagram of gyrostabilized telescope with VIP system. 


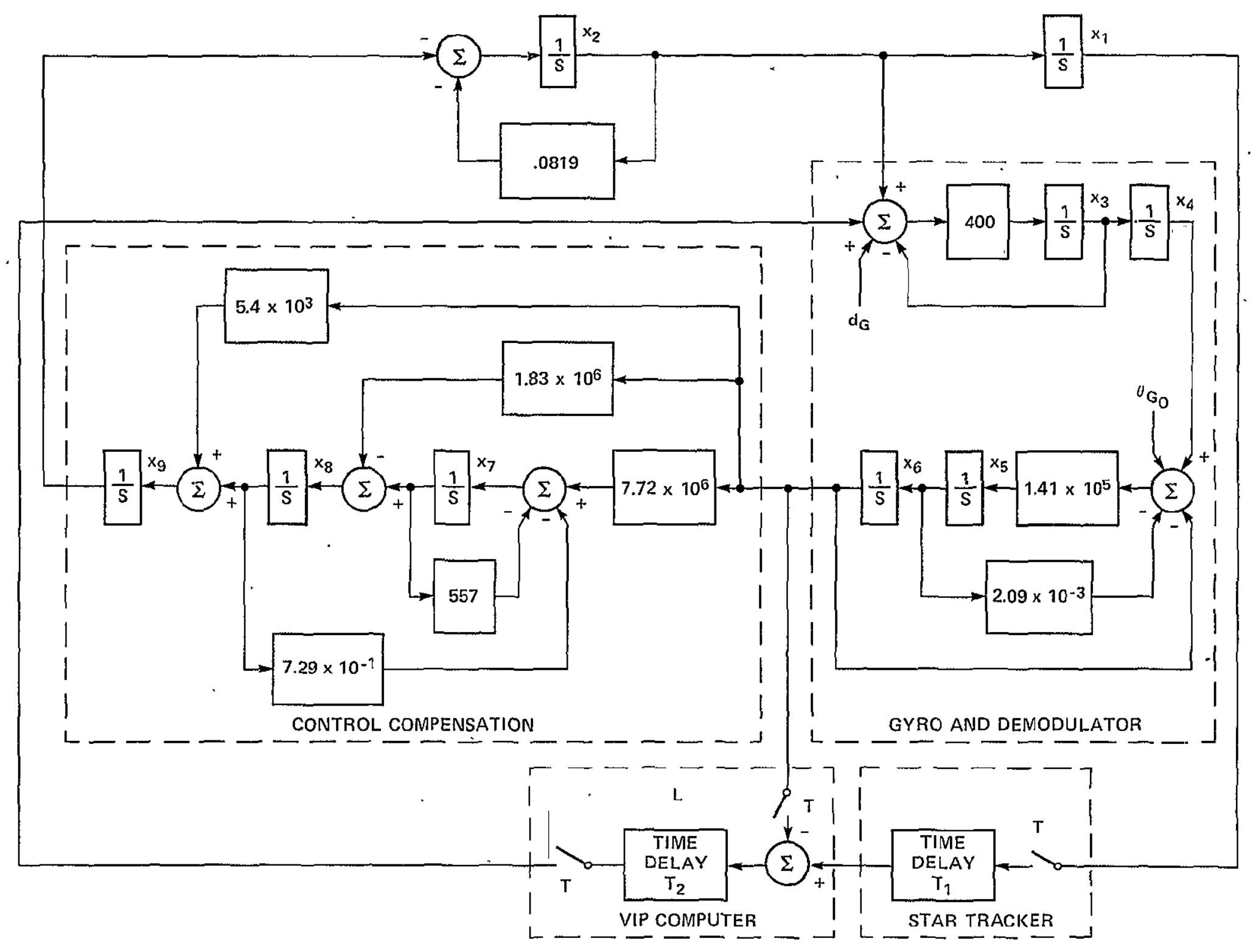

Figure 8.- State variable representation of gyrostabilized telescope with numerical values for AIROScope system. 


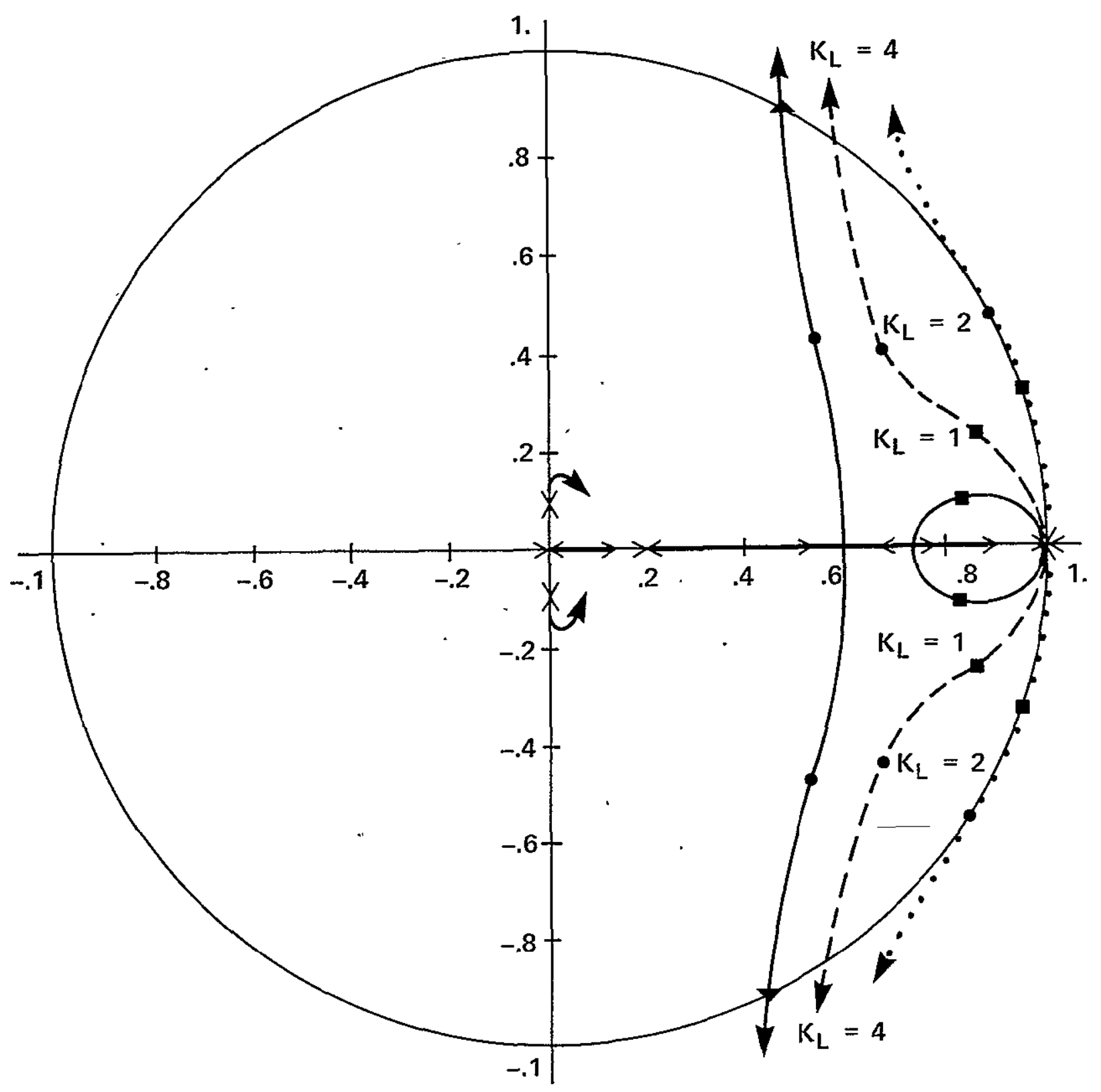

Figure 9.- Z-plane loci for $T_{1}=0.25 \mathrm{sec}$. Sample period $=0.25 \mathrm{sec}$, delay period $=0.25 \mathrm{sec}, \longrightarrow \mathrm{K}_{\mathrm{P}}=0.3, \cdots \mathrm{K}_{\mathrm{P}}=0.8, \cdots \mathrm{K}_{\mathrm{P}}=0.5$. 


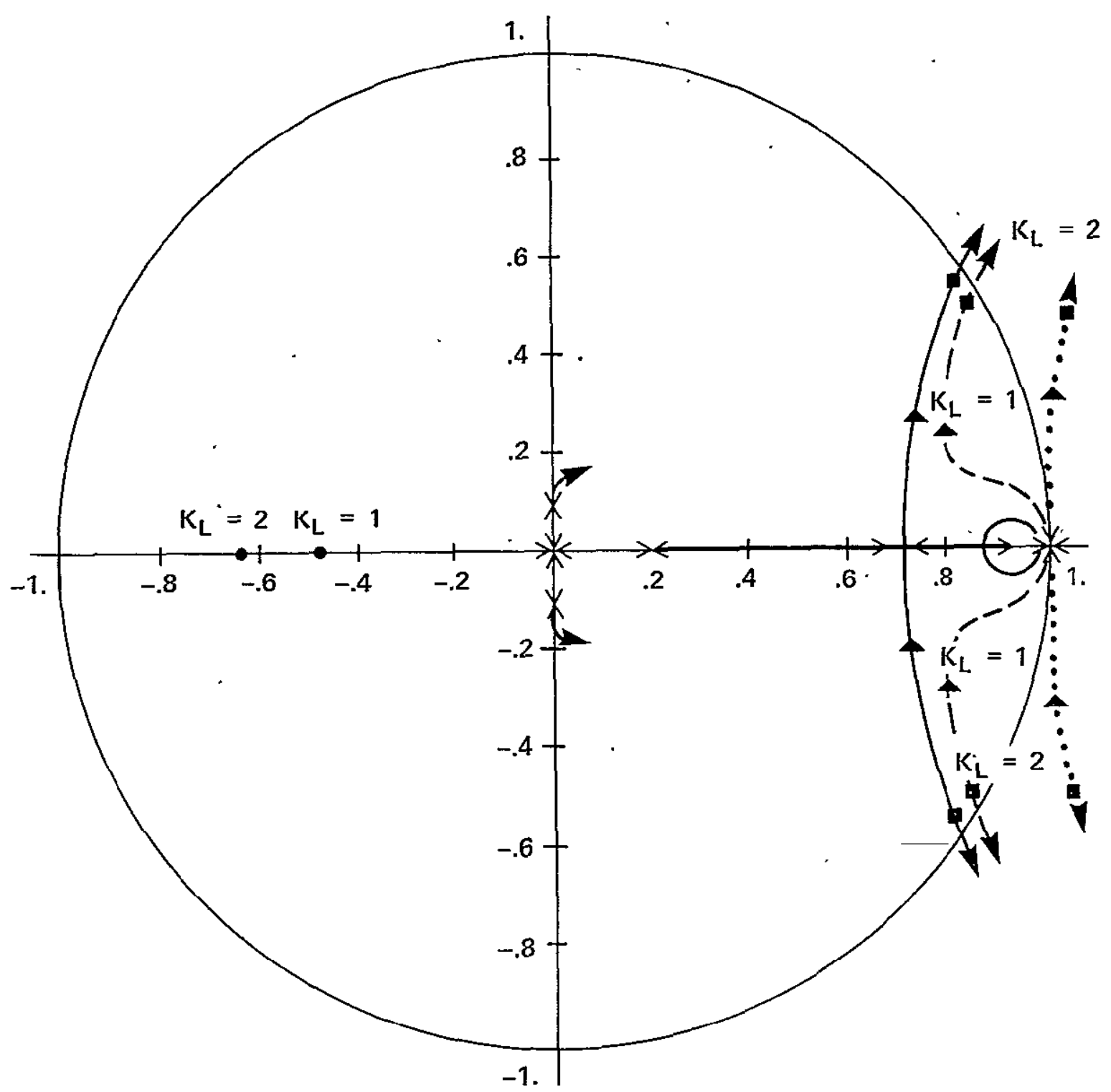

Figure 10. Z Z-plane" loci for $\mathrm{T}_{1}=0.50 \mathrm{sec}$. Sample perlod $=0.50 \mathrm{sec}$, delay period $=0.50 \mathrm{sec}, \longrightarrow \mathrm{K}_{\mathrm{P}}=0.95, \cdots \mathrm{K}_{\mathrm{P}}=0.9, \cdots \mathrm{K}_{\mathrm{P}}=0.5$. 


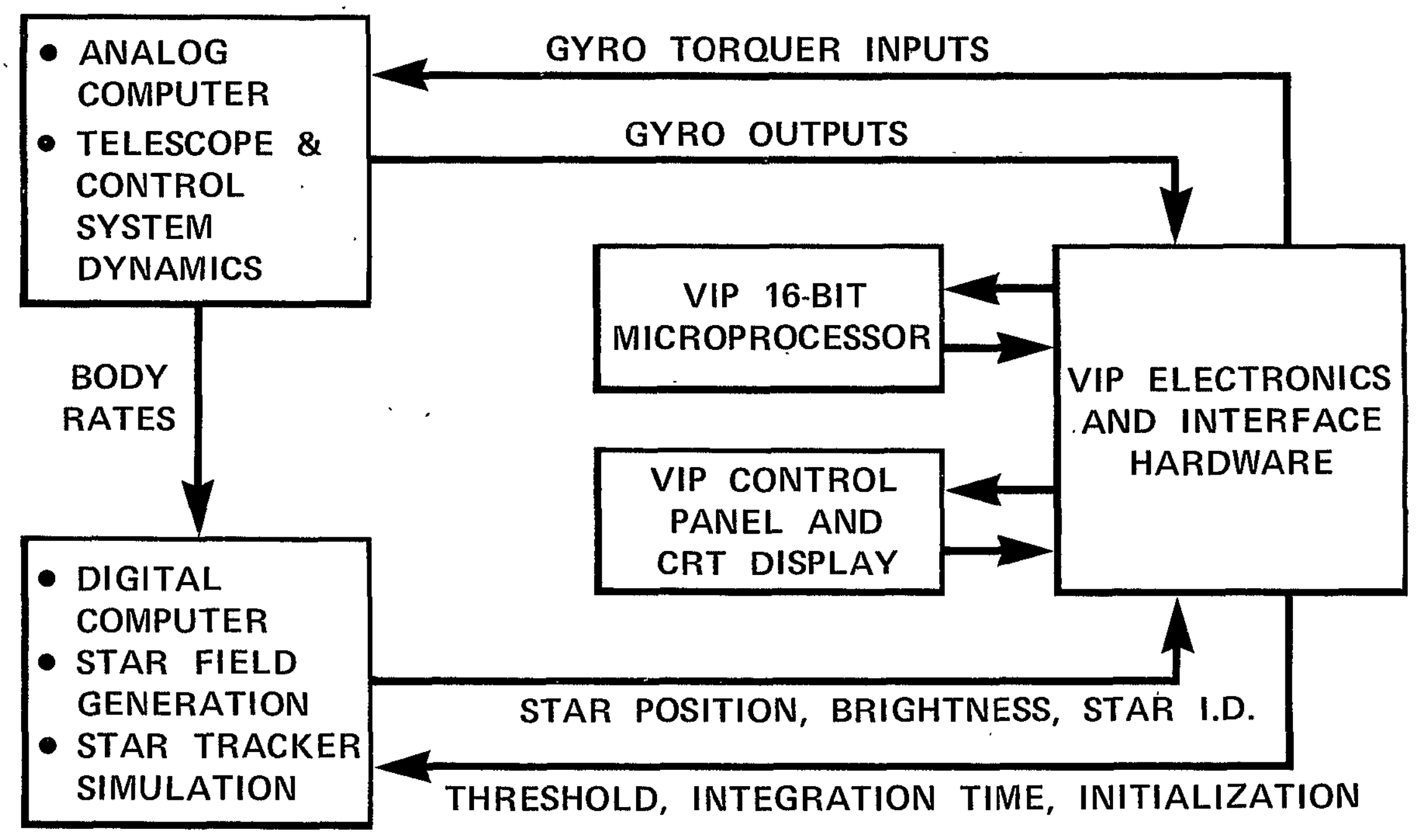

Figure 11.- Hybrid simulation block diagram. 


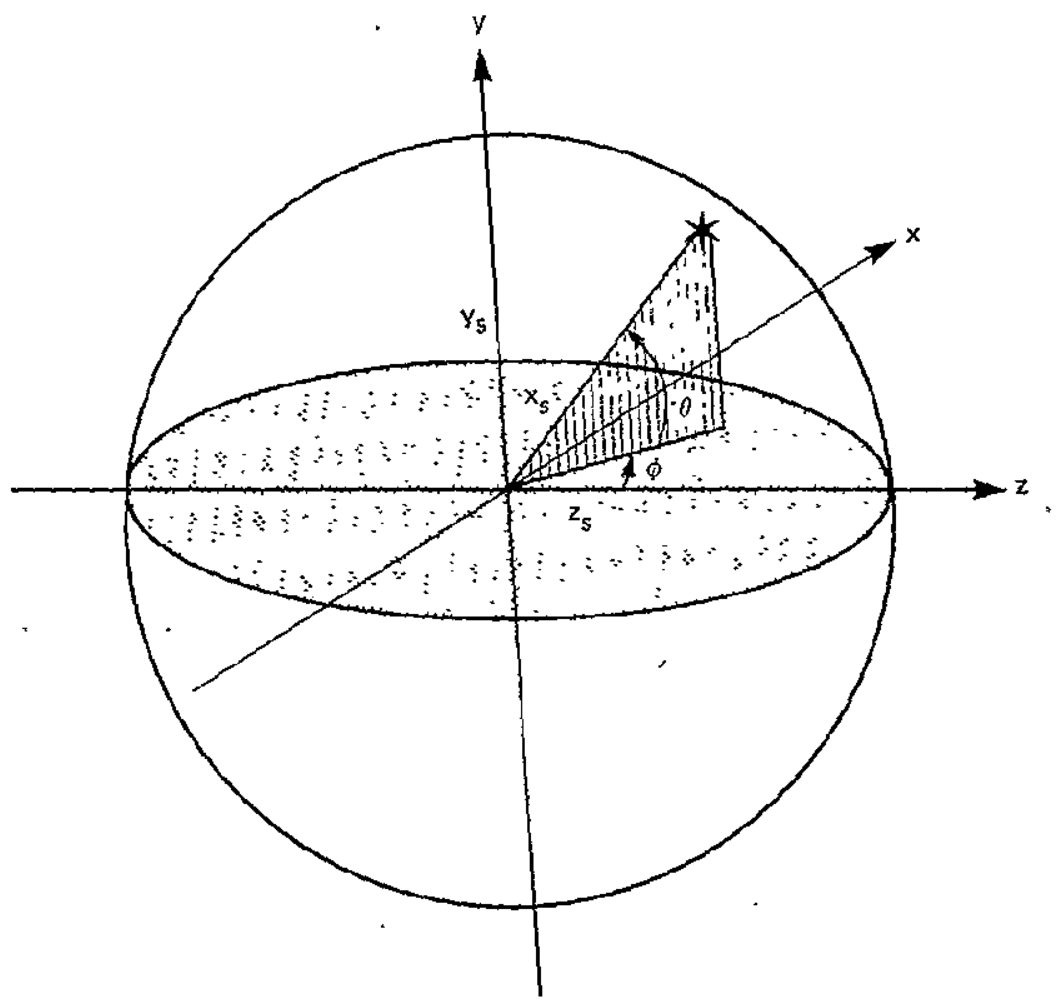

Figure 12. - HP-2114 star field coordinate system. 


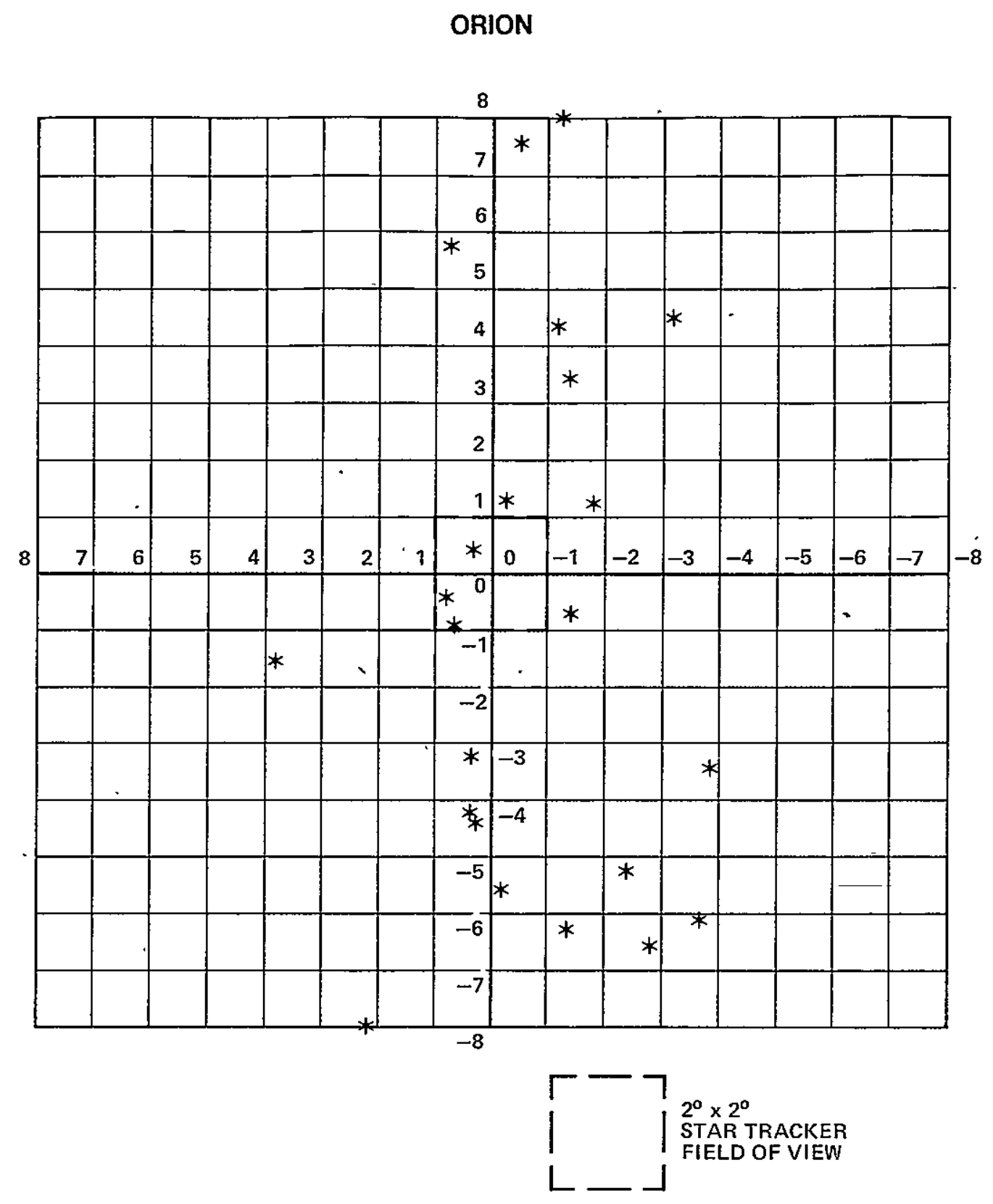

Figure 13.- Constellation Orion used in hybrid simulation. 


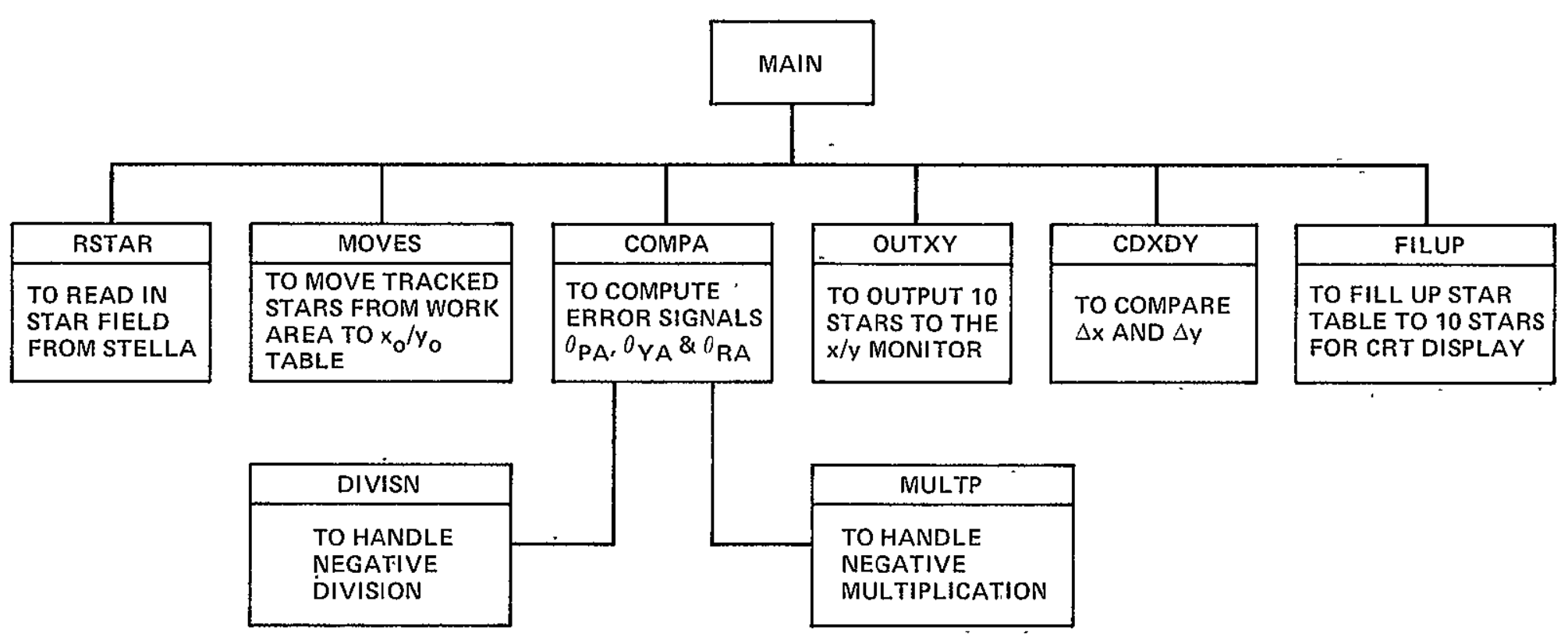

(a) HP-2114 software organization

Figure 14.- HP-2114 software organization and flow chart: 

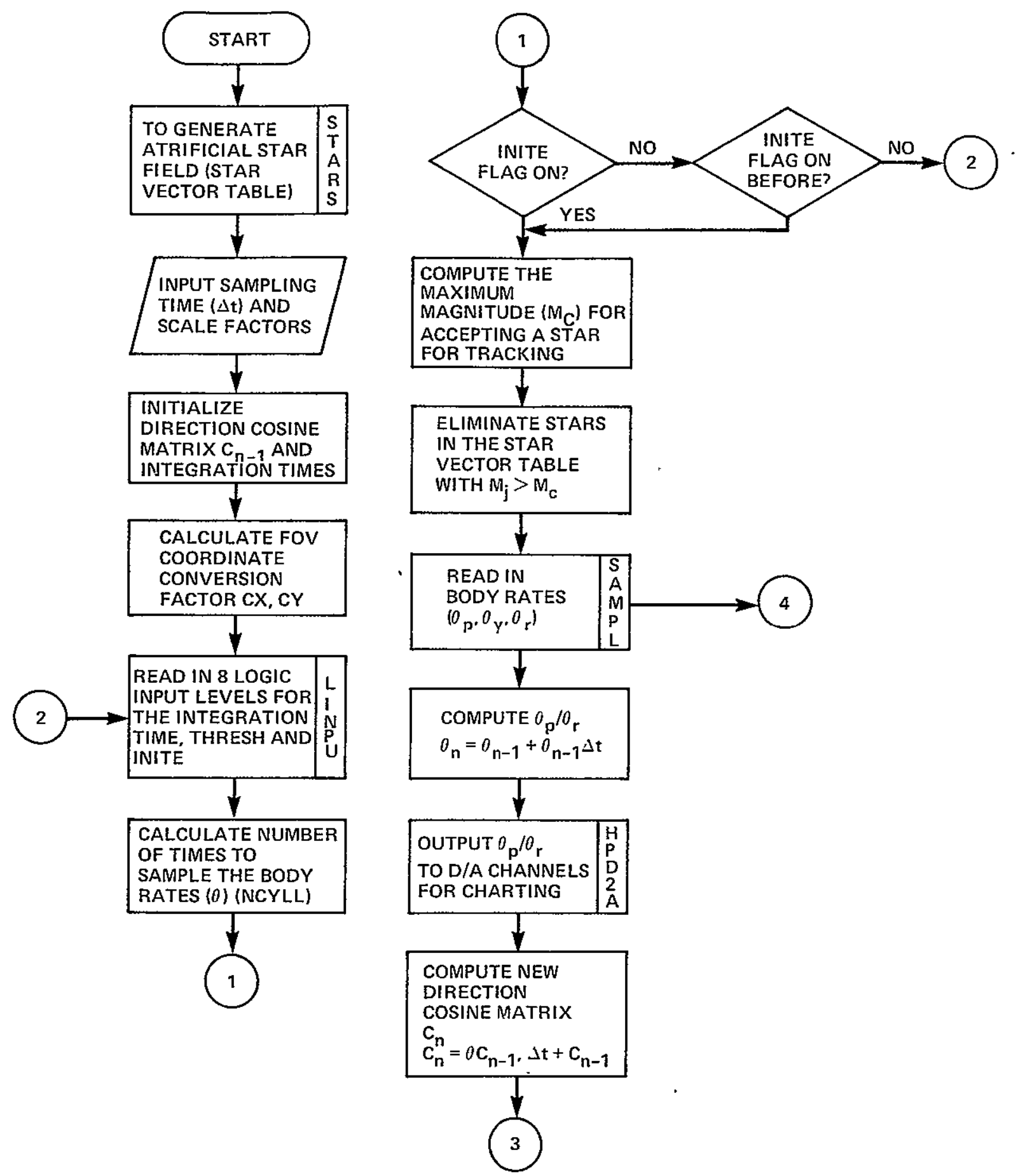

(b) HP-2114 software flow chart.

Figure 14.- Continued. 


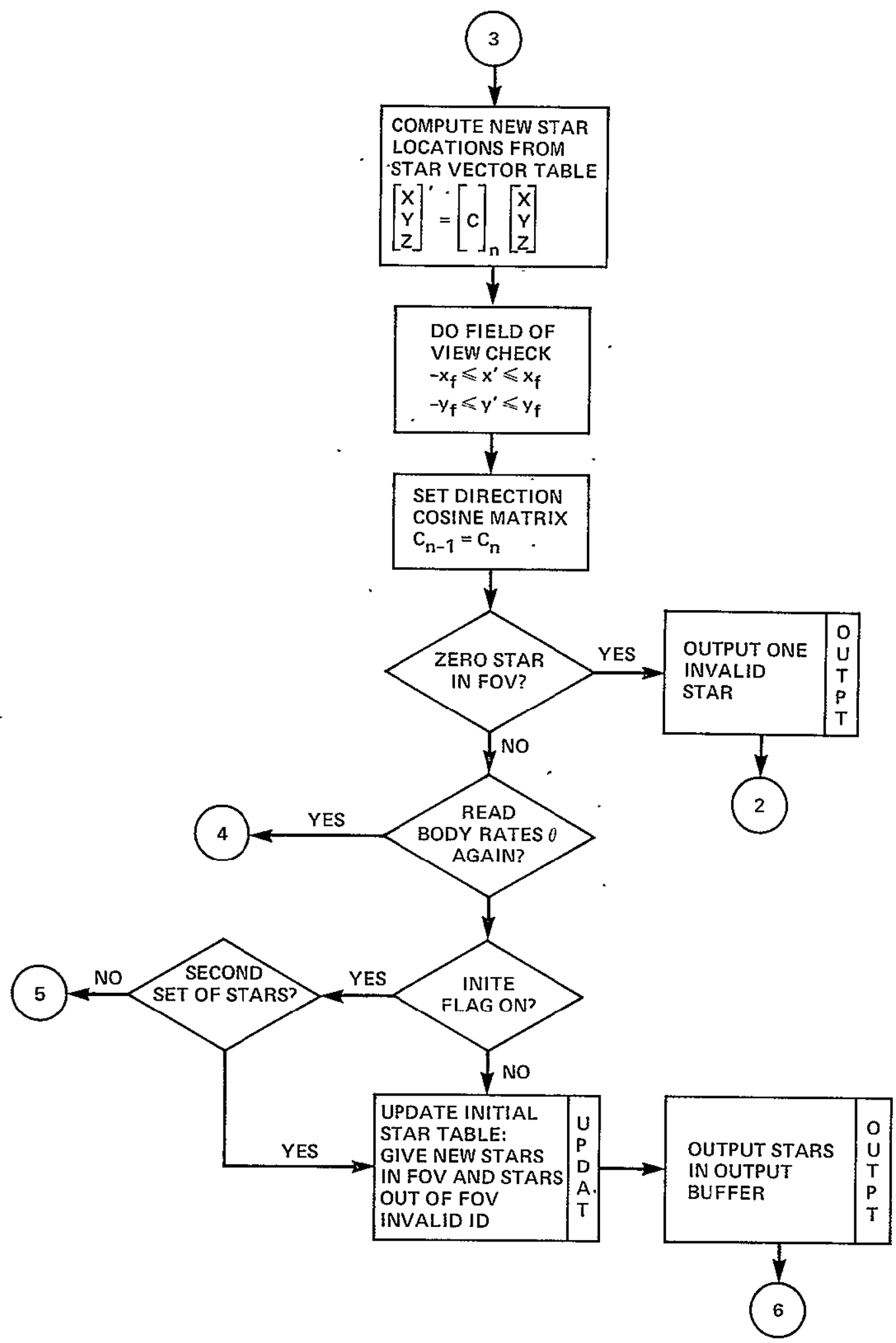

(c) HP-2114 software flow chart.

Figure 14.- Continued. 


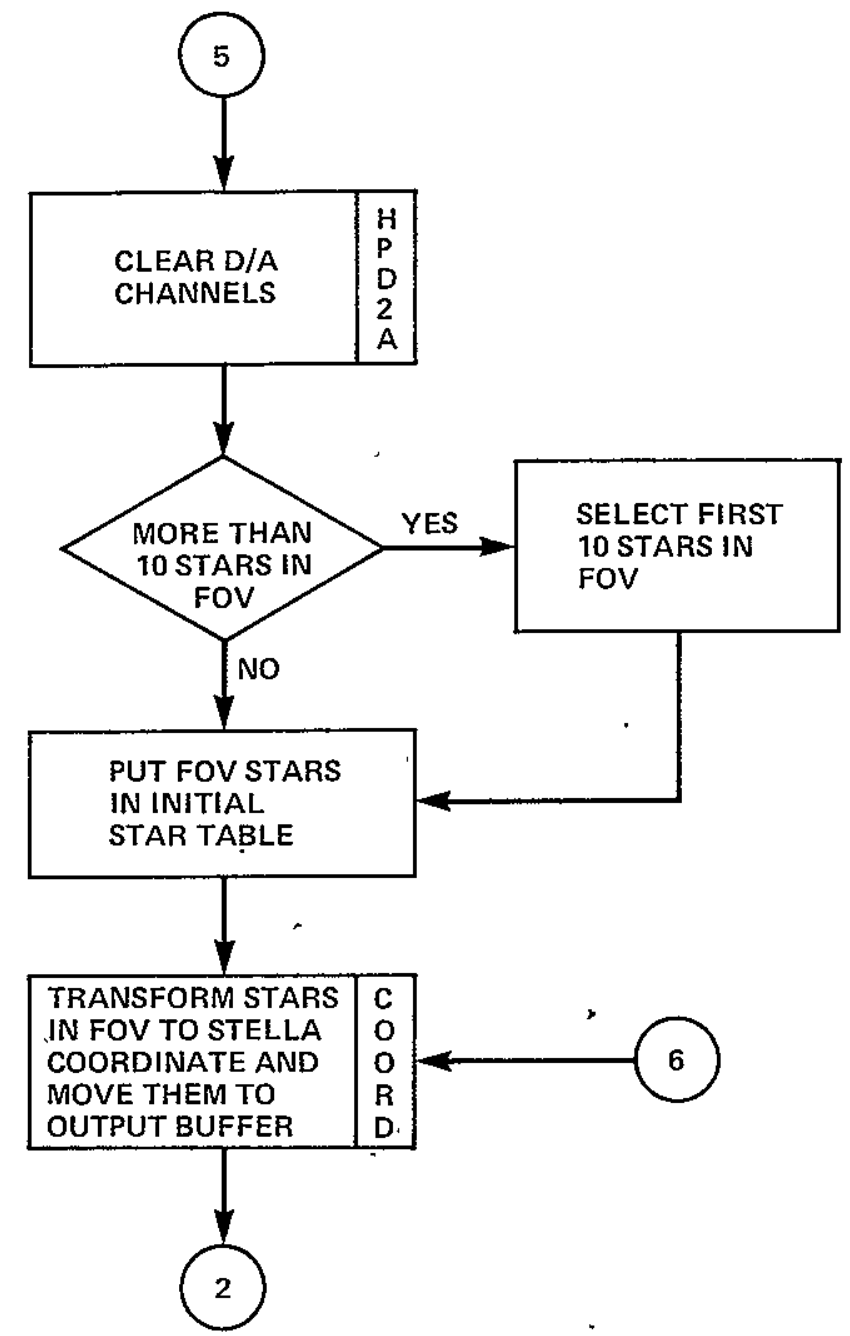

(d) HP-2114 software f1ow chart.

Figure 14.- Continued. 


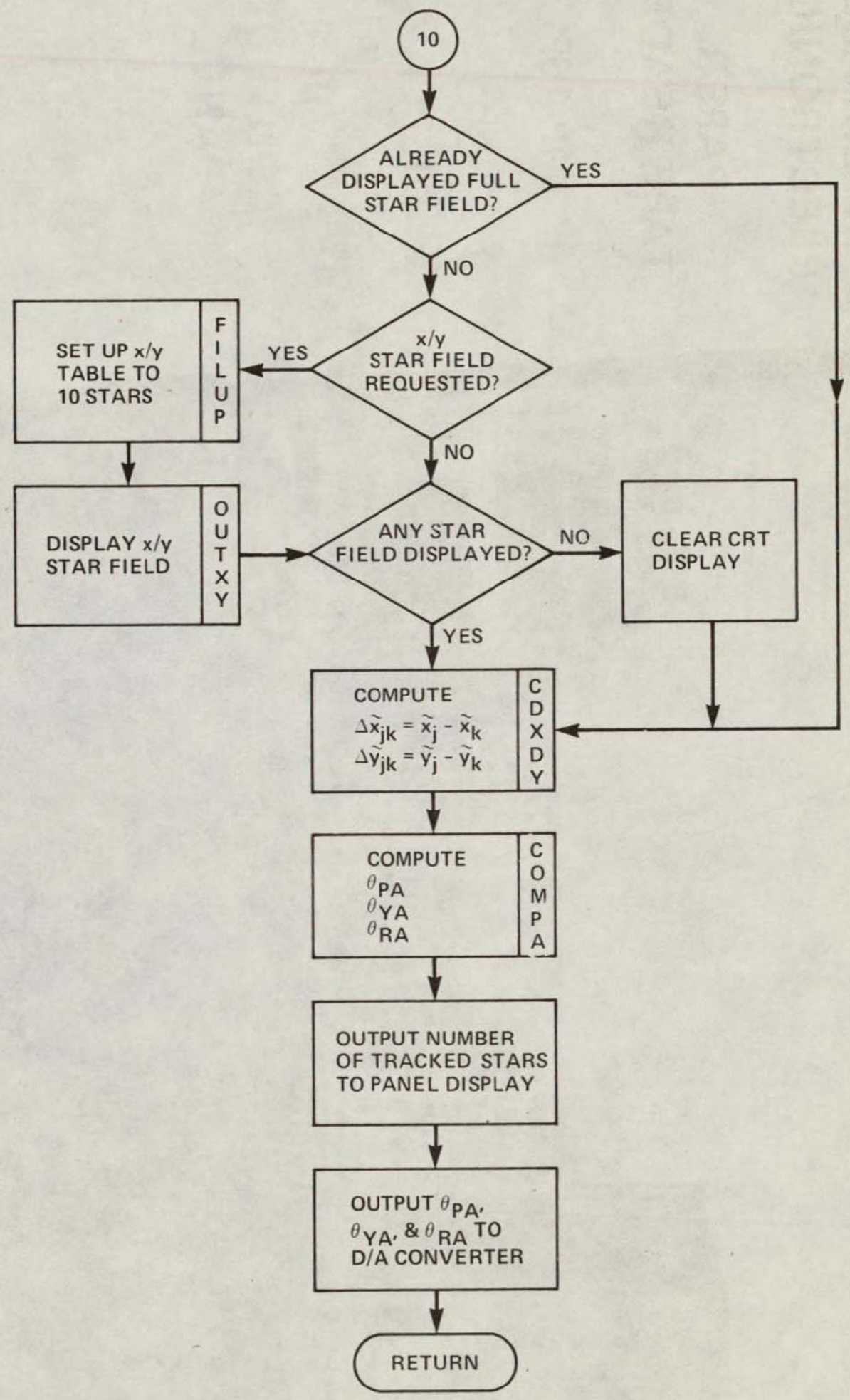

(e) HP-2114 software flow chart.

Figure 14.- Concluded. 
HP-2114

\section{DIGITAL COMPUTER}

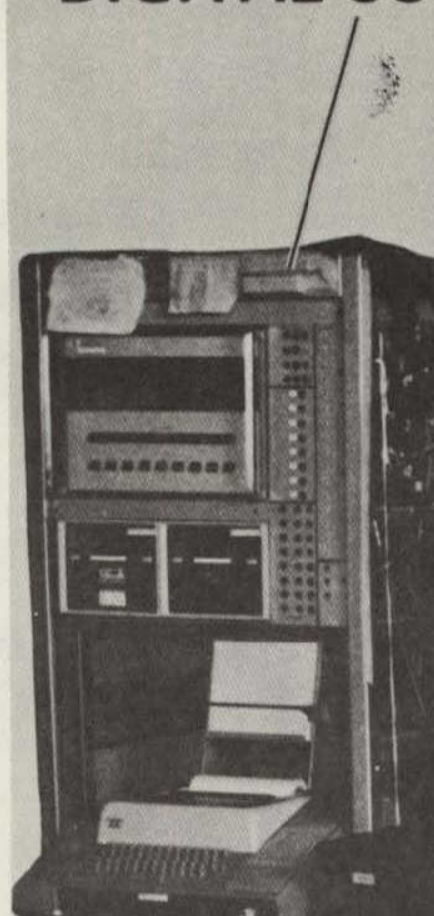

,
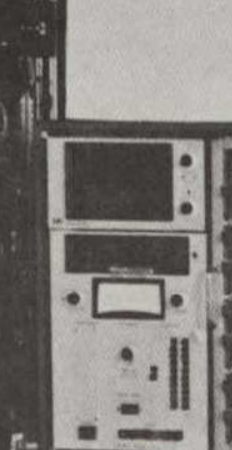

EAI TR-48 ANALOG COMPUTER

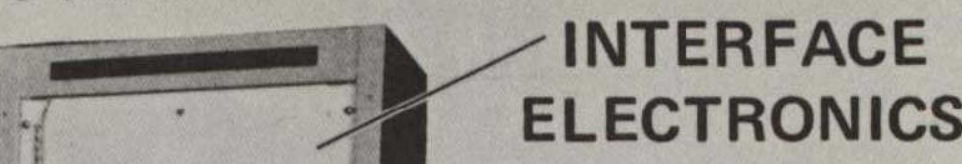

PAPER TAPE READER
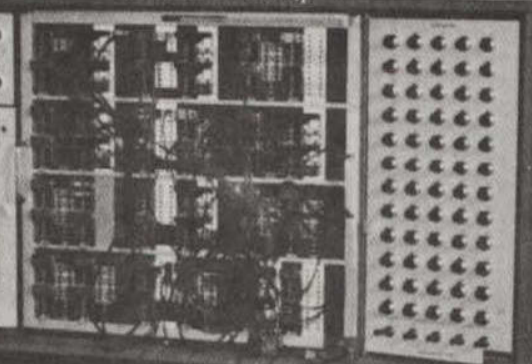

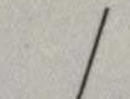

(1) , IIMP-16P CRT DISPLAY VIP CONTROL PANEL

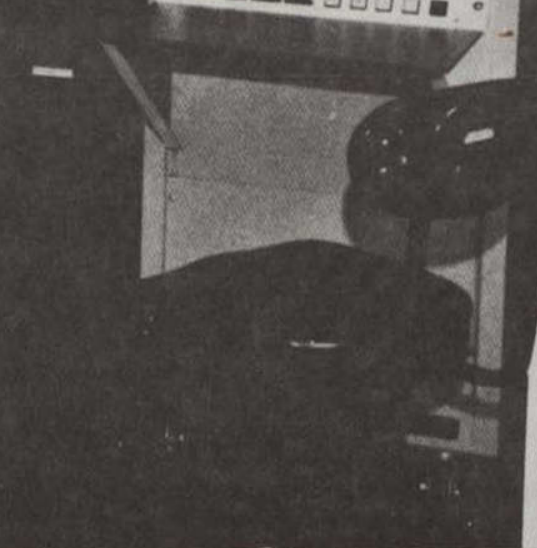

Figure 15.- Laboratory set-up for VIP hybrid simulation. 

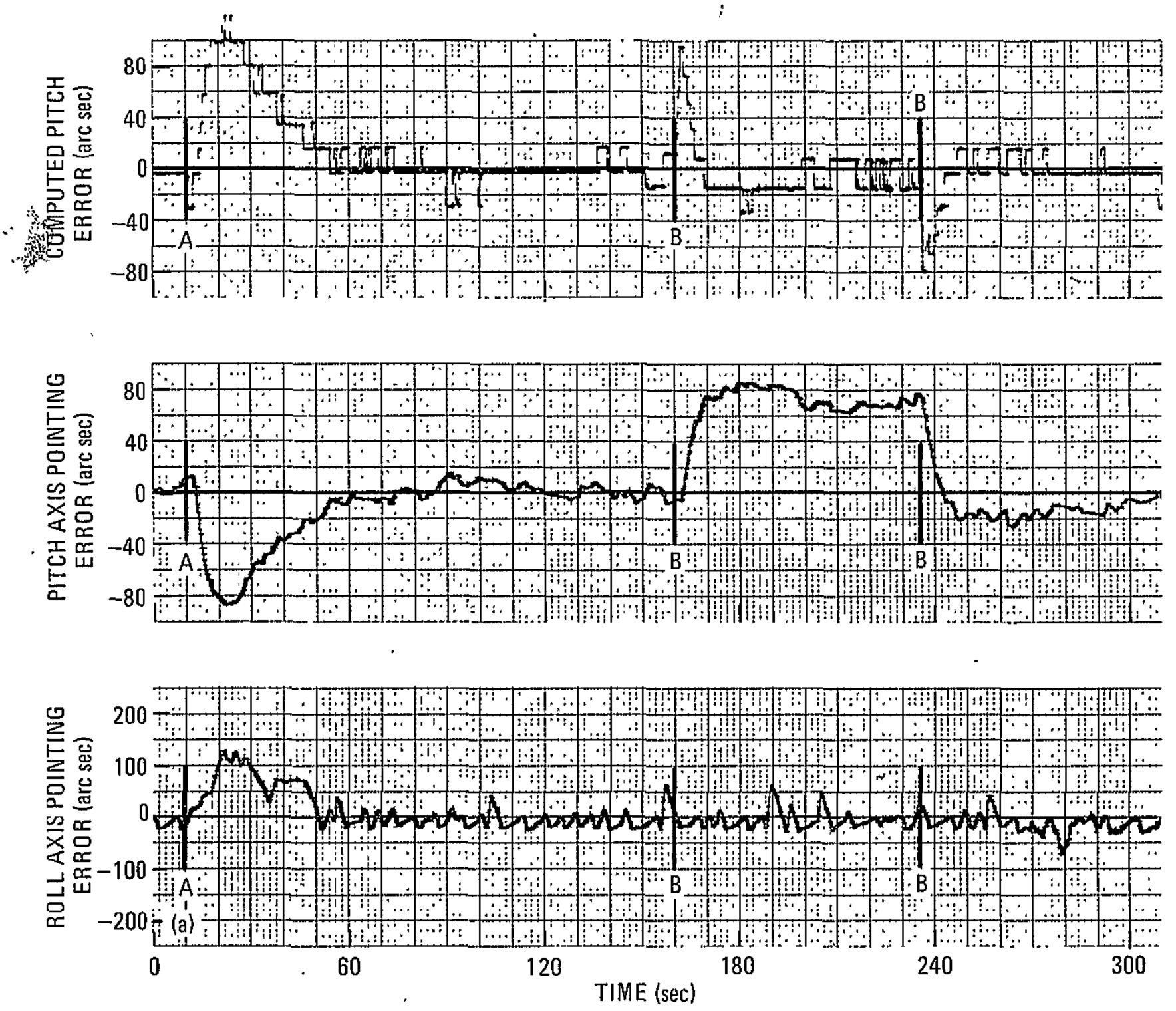

(a) Pitch and roll axis responses; note roll stability during pitch joystick command.

Figure 16.- VIP system performance from hybrid simulation. Sample time $=0.25$ sec, delay time $=0.25$ sec, field of view $=2^{\circ} \times 2^{\circ}$, tracked stars $=3$. A. $10^{\circ} / \mathrm{hr}$ step drift input to roll and pitch gyros.

B. Tovstick command in bitch. 

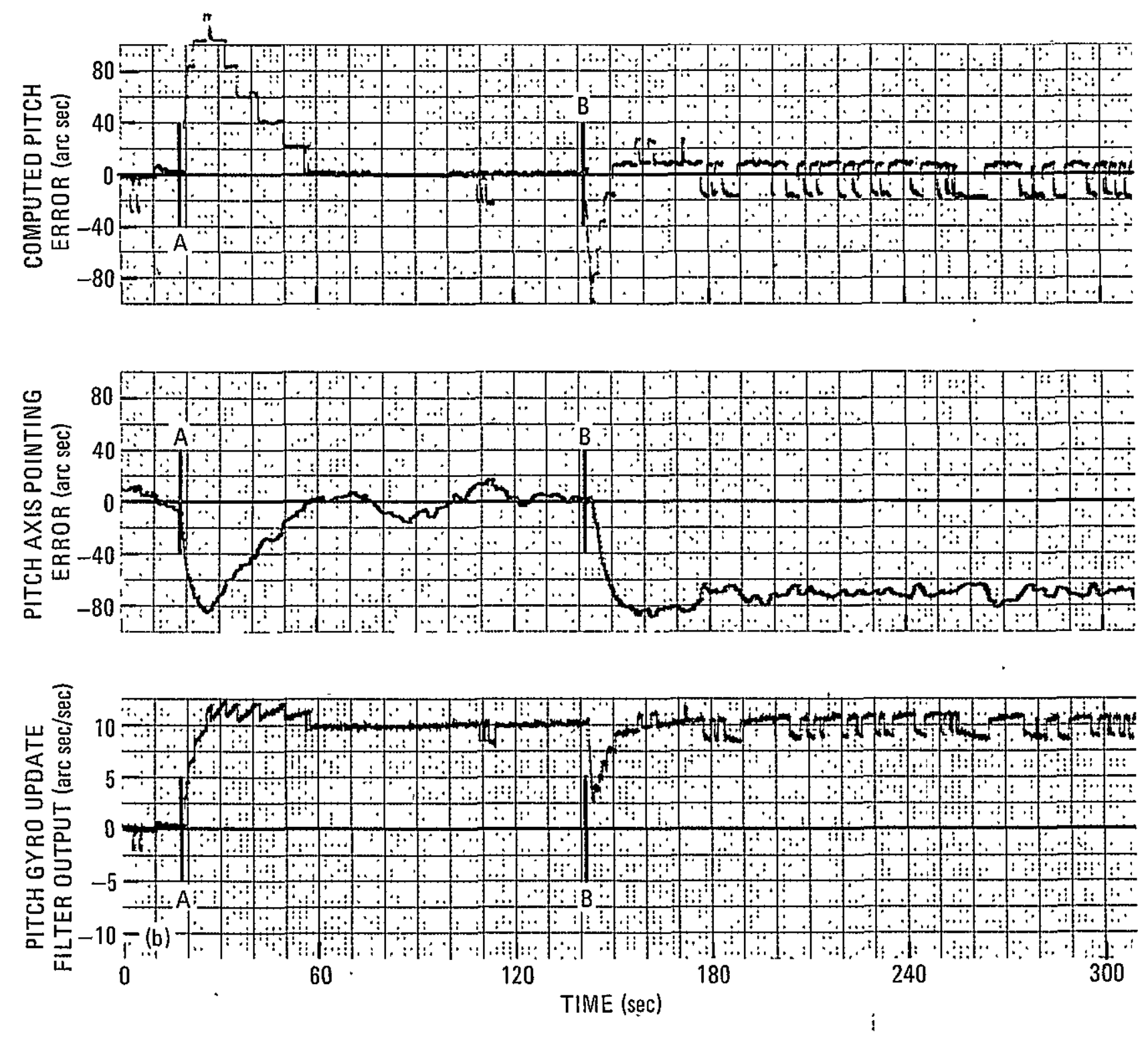

(b) Pitch axis filter response.

- Figure 16.- Concluded. 